Int. J. Dev. Biol. 59: 327-340 (2015)

doi: $10.1387 / \mathrm{ijdb} .150197 \mathrm{ml}$

\title{
Local and long-range endogenous resting potential gradients antagonistically regulate apoptosis and proliferation in the embryonic CNS
}

\author{
VAIBHAV P. PAI ${ }^{1}$, JOAN M. LEMIRE ${ }^{1}$, YING CHEN ${ }^{2}$, GUFA LIN ${ }^{2}$ and MICHAEL LEVIN ${ }^{*}, 1$ \\ ${ }^{1}$ Biology Department, and Center for Regenerative and Developmental Biology Tufts University, Medford, MA, USA \\ and ${ }^{2}$ Stem Cell Institute, University of Minnesota, Minneapolis, MN, USA
}

\begin{abstract}
Bioelectric signals, particularly transmembrane voltage potentials $\left(\mathrm{V}_{\text {mem }}\right)$, play an important role in large-scale patterning during embryonic development. Endogenous bioelectric gradients across tissues function as instructive factors during eye, brain, and other morphogenetic processes. An important and still poorly-understood aspect is the control of cell behaviors by the voltage states of distant cell groups. Here, experimental alteration of endogenous $V_{\text {mem }}$ was induced in Xenopus laevis embryos by misexpression of well-characterized ion channel mRNAs, a strategy often used to identify functional roles of $V_{\text {mem }}$ gradients during embryonic development and regeneration. Immunofluorescence analysis (for activated caspase 3 and phosphor-histone $\mathrm{H} 3 \mathrm{P}$ ) on embryonic sections was used to characterize apoptosis and proliferation. Disrupting local bioelectric signals (within the developing neural tube region) increased caspase 3 and decreased $\mathrm{H} 3 \mathrm{P}$ in the brain, resulting in brain mispatterning. Disrupting remote (ventral, non-neural region) bioelectric signals decreased caspase 3 and highly increased H3P within the brain, with normal brain patterning. Disrupting both the local and distant bioelectric signals produced antagonistic effects on caspase 3 and H3P. Thus, two components of bioelectric signals regulate apoptosisproliferation balance within the developing brain and spinal cord: local (developing neural tube region) and distant (ventral non-neural region). Together, the local and long-range bioelectric signals create a binary control system capable of fine-tuning apoptosis and proliferation with the brain and spinal cord to achieve correct pattern and size control. Our data suggest a roadmap for utilizing bioelectric state as a diagnostic modality and convenient intervention parameter for birth defects and degenerative disease states of the CNS.
\end{abstract}

KEY WORDS: $V_{\text {men }}$ non-cell-autonomous, apoptosis, proliferation, brain, bioelectricity

Introduction

A key question in developmental biology concerns the reliable self-assembly of the correct 3-dimentional morphology. The tight coordination of global patterning cues which control celllevel behaviors such as proliferation and apoptosis sculpt organs to acquire the correct size, shape, and orientation relative to each other during embryogenesis. Embryonic central nervous system (CNS) development is an ideal context in which to explore and understand the signals responsible for achieving the necessary target morphology. Developmental mispatterning is the cause of debilitating disorders, such as spina bifida (unclosed neural tube) (Copp and Greene, 2010, Detrait et al., 2005), anencephaly (small brain) (Copp and Greene, 2010, Detrait et al., 2005, Wallingford, 2006) and also increased susceptibility to degenerative disorders like Parkinson's, Alzheimer's, and autism (Doganli et al., 2013, Goldman et al., 2013, Pratt and Khakhalin, 2013). Hence, understanding how large-scale patterning of the brain normally occurs is a pre-requisite for major biomedical breakthroughs addressing birth defects and repairing injuries.

Organ size and morphology, especially in relation to neighboring tissues and the organism as a whole, are precisely controlled in all

Abbreviations used in this paper: CNS, Central Nervous System; GJCs, gap junctional communication; $\mathrm{V}_{\text {mem }}$, transmembrane voltage potential.

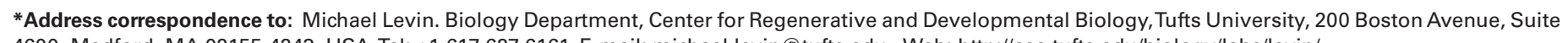
4600, Medford, MA 02155-4243, USA.Tel: +1-617-627-6161. E-mail: michael.levin@tufts.edu - Web: http://ase.tufts.edu/biology/labs/levin/ 
forms of multicellular life from plants to animals (Stanger, 2008a, Stanger, 2008b). Appropriate organ size is achieved through regulation of proliferation, cell death and remodeling especially during development (Joseph and Hermanson, 2010, Stanger, 2008a, Stanger, 2008b). During CNS development, a precisely orchestrated control of cell proliferation, cell death and cell differentiation results in expansion of the neural progenitor populations ultimately leading to formation of the complex neural structures (brain and spinal cord) from the pseudo-stratified neuroepithelium (Joseph and Hermanson, 2010, Stanger, 2008a, Stanger, 2008b). Gene regulatory networks (Harvey and Hariharan, 2012, Zhao et al., 2011) and physical forces (Stanger, 2008b, Thompson, 1942) have been shown to regulate this complex process. Here we characterize the role of a novel player: transmembrane voltage potential $\left(\mathrm{V}_{\text {mem }}\right)$ patterns across the developing Xenopus embryo, in regulating apoptosis and proliferation within the developing brain.

Every cell (not just excitable neurons and muscle cells) has a characteristic $\left(V_{m e m}\right)$ across their plasma membrane, which is a result of combination of all ion fluxes through channels and pumps. Spatio-temporal gradients of $\mathrm{V}_{\text {mem }}$ across groups of cells serve as instructive bioelectric signals (Levin, 2013b, Levin, 2014b, Mustard and Levin, 2014). Such patterns of resting potential within living tissues (bioelectric signals) have been studied in the context of their roles in cell migration and wound healing (Cao et al., 2013, Jaffe, 1979, McCaig et al., 2005, Richard B. Borgens, 1989, Zhao et al., 2006) and have long been proposed to direct growth and form in vivo (Burr, 1932). Bioelectric signals are implicated in vertebrate appendage regeneration (Adams et al., 2007, Tseng et al., 2010), cancer initiation and metastasis (Binggeli and Weinstein, 1986b, Blackiston et al., 2011, Brackenbury, 2012, Chernet et al., 2014, Chernet and Levin, 2013b, Chernet and Levin, 2014, Lobikin et al., 2012b), left-right patterning (Aw et al., 2008, Aw et al., 2010, Levin et al., 2002), planarian head induction (Beane et al., 2011, Beane et al., 2013, Marsh and Beams, 1947), and eye and brain formation (Nuckels et al., 2009, Pai et al., 2015, Pai et al., 2012a, Pai et al., 2012b). Thus bioelectric signals have been shown to be important regulators of large-scale patterning and tissue and organ identity (Levin, 2009, Levin, 2013a, Levin and Stevenson, 2012, Tseng and Levin, 2013a).

In the brain, electrical activity within neural precursors shapes neuronal connections within the developing CNS (Borodinsky et al., 2004, Deisseroth et al., 2004, Swapna and Borodinsky, 2012). Previously, bioelectric signals have been shown to be important determinants of nascent brain morphology (Pai et al., 2015 J. Neuro). Here, we analyze the mechanism of this instructive interaction, by characterizing the role of bioelectrical gradient patterns in regulating apoptosis and cell proliferation during early embryonic CNS (brain and spinal cord) development. The Xenopus laevis embryo is an excellent model for studying CNS development (Pratt and Khakhalin, 2013) and facilitates cell-level dissection of bioelectrical signals in embryonic developmental events. Our data indicate that bioelectrical signals regulate both apoptosis and proliferation in the developing CNS. Interestingly, both local (within the developing neural tube) bioelectric cell states and distant (ventral) bioelectric cell states are involved in controlling the amount and location of both apoptosis and proliferation within the CNS. Importantly, the local and distant bioelectric signals function counter to each other, forming a binary control system that can fine-tune the extent of apoptosis and proliferation within the CNS to tightly regulate tis- sue size in vivo. These results shed light on a new endogenous developmental mechanism and suggest strategies for modulating growth and form in applications targeting birth defects and degenerative disease states.

\section{Results}

\section{Brain development disrupted by local $V_{\text {mem }}$ perturbation is reversed by long-distance $V_{\text {mem }}$ signaling}

Adynamic endogenous bioelectrical prepattern drives craniofacial development in Xenopus (Vandenberg et al., 2011). The central neural plate cells exhibit a strong hyperpolarization as the neural plate folds to form the neural tube (Pai et al., 2015, Pai et al., 2012a), and forced deviation from this endogenous $V_{\text {mem }}$ pattern causes disruption of endogenous brain development (Pai et al., 2015, Pai et al., 2012a). Given the role of local $V_{\text {mem }}$ states (bioelectric signals in the developing brain region) in shaping brain development (Pai et al., 2015, Pai et al., 2012a), and the importance for coordinating brain size with other anatomical features in vivo, we asked whether non-local $V_{\text {mem }}$ distributions (bioelectric states of cells far away from the developing brain) might affect endogenous brain development across long distances. Experimental alteration of $\mathrm{V}_{\text {mem }}$ was induced by misexpression of well-characterized ion channel mRNAs, a strategy often used to identify functional roles of $\mathrm{V}_{\text {mem }}$ during embryonic development and regeneration (Adams and Levin, 2006, Aw et al., 2008, Levin et al., 2002, Pai et al., 2012a, Pai and Levin, 2014, Pai et al., 2012b, Perathoner et al., 2014, Vandenberg et al., 2011). We specifically altered $\mathrm{V}_{\text {mem }}$ of cells within relevant (local and/or nonlocal) regions by injecting mRNAs encoding the hyperpolarizing channel Kv1.5[voltage-gated potassium channel(Strutz-Seebohm et al., 2007)]. The effect of introducing this ion channel mRNA on $\mathrm{V}_{\text {mem }}$ of cranial and other cells in the embryo has previously been characterized at stages 10-21 using the voltage reporter dyes and electrophysiology (Pai et al., 2015, Pai et al., 2012a), confirming its ability to efficiently hyperpolarize expressing cells. In vivo, Kv1.5 misexpression eliminates the endogenous $V_{\text {mem }}$ differences and spatial gradients within the group of channel expressing cells, driving expressing cells to $\sim-58 \mathrm{mV}$, from an endogenous polarization level of $\sim-20 \mathrm{mV}$ or $\sim-50 \mathrm{mV}$ of cells outside and inside the prospective brain respectively.

Kv1.5 mRNA (titrated to the lowest levels that produced brain phenotypes) was injected into the dorsal two cells of four-celled embryos [the blastomeres from which neural tissue is derived - local (Moody, 1987)], or the ventral two cells of four-celled embryos [blastomere which do not contribute to neural tissue - non-local; (Moody, 1987)], or both dorsal and ventral blastomeres (Fig. 1A). As previously documented by us and others, we saw no sign of general toxicity or non-specific ill health [midline patterning, scale, proportions and overall growth were normal; (Adams and Levin, 2013, Blackiston et al., 2011, Pai et al., 2012a)(Pai et al., 2015, Pai et al., 2012a)]. Uninjected and water-injected embryos served as controls. To document even subtle changes in brain tissue morphology, we used a transgenic frog line PNTub-GFP (Lin et al., 2012, Marsh-Armstrong et al., 1999), where the neural tubulin promoter drives GFP expression giving rise to tadpoles with GFP-labeled CNS tissue (Fig. 1B). This allowed clear visualization of all Xenopus brain and spinal structures, and any deviation from normal patterning.

To determine whether ventral (non-neural) regions' $V_{\text {mem }}$ patterns could affect dorsal neural tissue development, Kv1.5-injected (dor- 
sal, ventral, or both) embryos were allowed to develop to stage 45, and brain morphology was evaluated (Fig. 1). Control tadpoles had intact anterior neural tissue with well-formed nostrils, olfactory bulbs/ forebrain, midbrain, hindbrain and spinal cord (Fig. 1Bi; (Pratt and Khakhalin, 2013). As expected (Pai et al., 2015, Pai et al., 2012a), introduction of $\sim 3-4 \mathrm{ng} K v 1.5$ mRNA in the dorsal blastomeres caused high incidence of disrupted endogenous brain tissue forma-

\section{A}

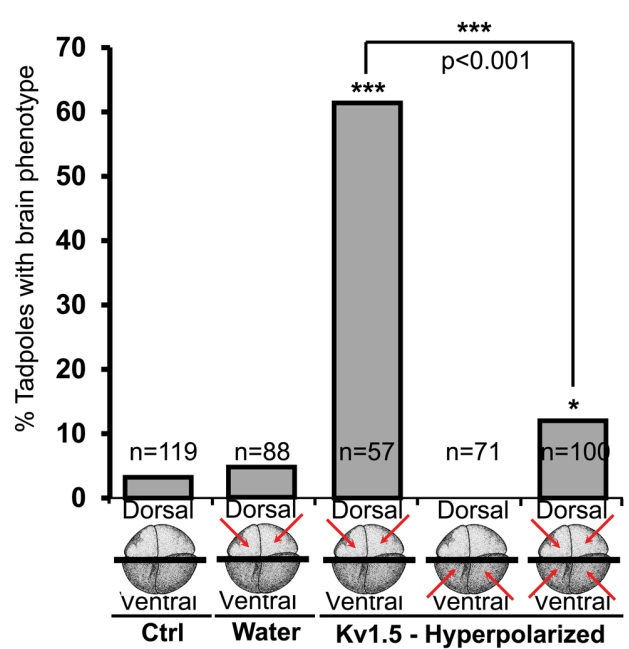

B
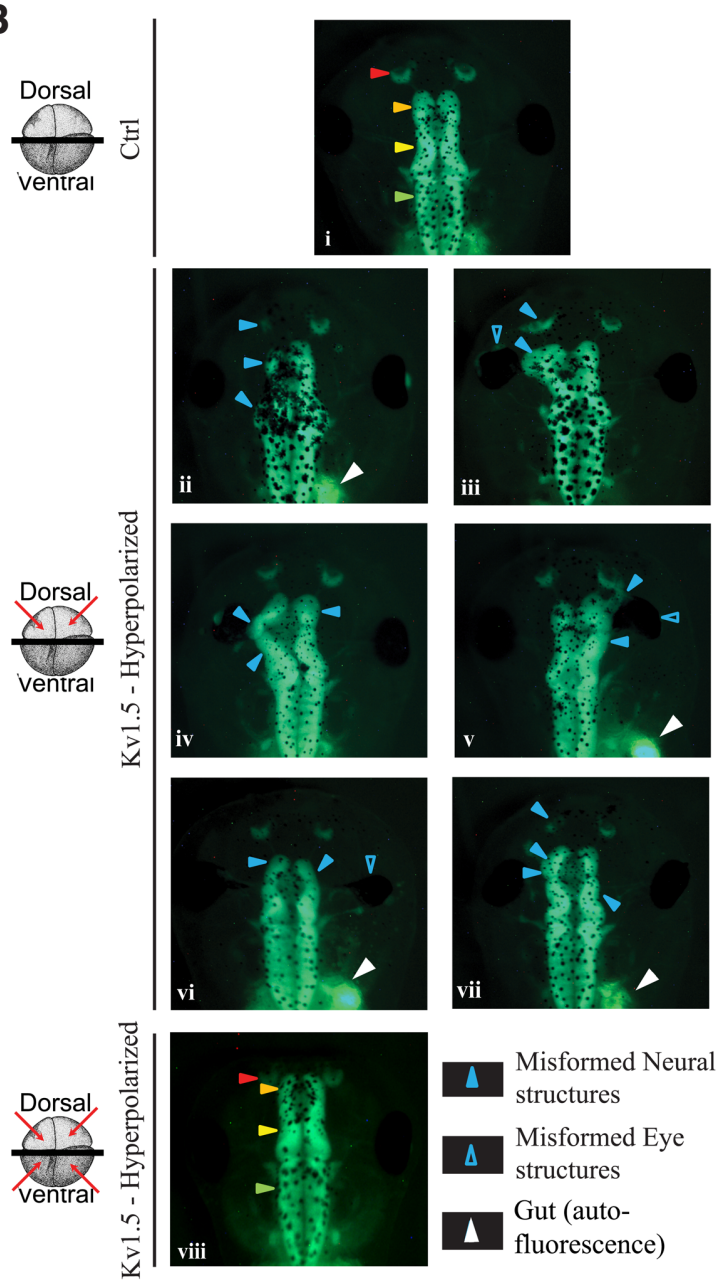

Misformed Neural structures Misformed Eye structures Gut (autofluorescence) tion ( $60 \%$; Fig. 1A). The phenotypes included: absence of nostrils, olfactory bulbs, and forebrain, with severely malformed midbrain (Fig. 1Bii-vii). Eye development was also affected, resulting in incompletely formed eyes, eyes fused to the brain and pigmented optic nerves (Fig. 1Bii-vii). However, because eye development is dependent on proper neural development (Fuhrmann, Harada et al., 2007, Zuber, 2010), it is likely that the eye defects may be a secondary manifestation of brain defects. Ventral Kv1.5 injections alone reduced (albeit non-significantly) the background incidence of brain phenotype in un-manipulated controls (Fig. 1A).

Interestingly, ventral $K v 1.5$ mRNA injections resulted in near complete prevention ( $10 \%$; Fig. 1A) of brain phenotypes induced by concomitant dorsal Kv1.5injections. These embryos, which were injected in both - dorsal and ventral blastomeres - show normal brain morphology similar to that of controls, with distinct nostrils, well-formed olfactory bulbs/forebrain, midbrain and hindbrain (Fig. 1Bviii). These data show that brain mispatterning caused by hyperpolarizing local (dorsal) region can be rescued/prevented by hyperpolarizing the distant (ventral) cells. These data confirm the importance of resting potentials for brain development, and demonstrates that the relevant $\mathrm{V}_{\text {mem }}$ is not merely that of the cells that actually form the brain: the bioelectric state of cells on the opposite side of the body matters as well, and indeed is able to completely rescue defects in local bioelectric state. The rescue rules out simple toxicity of channel misexpression as the explanation for the brain defect phenotype, and reveals a long-range signaling component to brain development.

\section{Both local and distant $V_{\text {mem }}$ signals regulate apoptosis in the developing brain}

What common cellular level processes could be regulated by the local and distant $\mathrm{V}_{\text {mem }}$ patterns to control embryonic brain development? The extent and pattern of apoptosis is a major factor that

Fig. 1. Kv1.5 mediated local $\mathrm{V}_{\text {mem }}$ perturbation disrupts endogenous brain development. (A) Quantification of tadpoles with brain phenotypes upon microinjections of hyperpolarizing Kv1.5 ion channel mRNA in the indicated cells (red arrows) of the four cells Xenopus embryo. A high incidence of misformed brain is observed in dorsal injections in comparison to uninjected controls. A $\chi^{2}$ analysis was performed. The dorsally injected embryos were significantly different from the controls (*** $p<0.001)$. Ventral injections were not significantly different from controls. Injections in both dorsal and ventral regions showed decrease in misformed brain in comparison to dorsal injections alone. A t-test showed both dorsally and ventrally co-injected embryos were significantly different from the dorsally injected embryos ( ${ }^{* *} p<0.001$ ). (B) Control (uninjected) and $\mathrm{Hy}$ perpolarizing Kv1.5 mRNA injected (red arrows indicate injected cells) in 4-cell PNTub::GFP transgenic Xenopus embryos. (i) Stage 45 PNTub::GFP controls show GFP fluorescence in the neural tissue. The uninjected control tadpoles show well-formed anterior neural tissues. Control tadpoles show well-formed anterior neural tissue with red arrowheads indicating nostrils, orange arrowheads indicating forebrain/olfactory bulbs, yellow arrowheads indicating mid-brain and green arrowheads indicating hindbrain. (ii-vii) Stage 45 PNTUb::GFP transgenic tadpoles dorsally injected with hyperpolarizing channe/ Kv1.5 mRNA. Solid Blue arrowheads indicate severely malformed midbrain and forebrain. Empty blue arrows indicate eyes which are also found to be malformed or absent. (viii) Stage 45 PNTub::GFP transgenic tadpoles injected with hyperpolarizing channe/ Kv1.5 mRNA in both dorsal and ventral blastomeres showing brain structure similar to the uninjected controls (i). Gut (white arrowhead) is also autofluorescent in the same spectrum. 
regulates tissue sculpting and tissue boundaries in developmental organ formation (Arya and White, 2015, Cowan and Roskams, 2004, Joseph and Hermanson, 2010, Miura, 2011, Nonomura et al., 2013, Perez-Garijo et al., 2013). Importantly, apoptosis is now known to be required for specific morphogenetic events such as regeneration (Bergmann and Steller, 2010, Chera et al., 2009, Tseng et al., 2007) - it is a constructive process, not merely a sign of ill health. Hence, we analyzed apoptosis within the developing brain of $\mathrm{V}_{\text {mem }}$-perturbed (hyperpolarized by Kv1.5 mRNA injection) Xenopus embryos, by immunostaining transverse sections through developing brain with an activated caspase 3 apoptosis marker (Porter and Janicke, 1999). Sections anterior/through the eye were used for analysis of developing brain tissue. In control (uninjected) embryos, active caspase 3 was found intermittently and sparsely distributed throughout the developing brain tissue (Fig. 2Ai). This pattern of apoptosis in the developing brain is consistent with previous reports of a low background of apoptosis involved in proper brain development (Chan et al., 2002, Nonomura et al., 2013, Rakic and Zecevic, 2000). Embryos injected with Kv1.5 (hyperpolarizing) mRNA into the dorsal two cells at four-cell stage
A
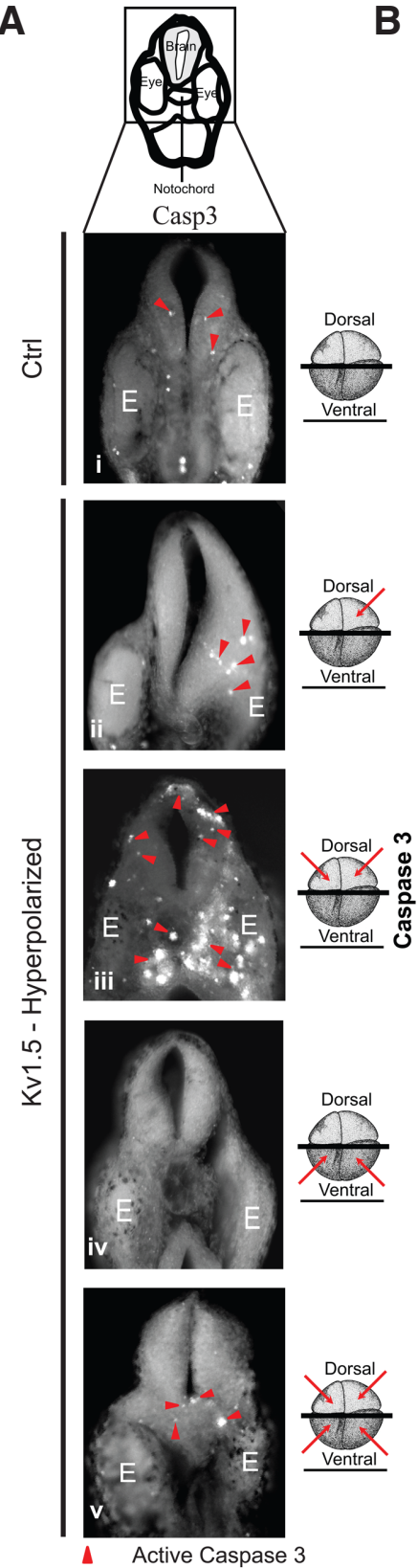

B Active Caspase3 in Brain

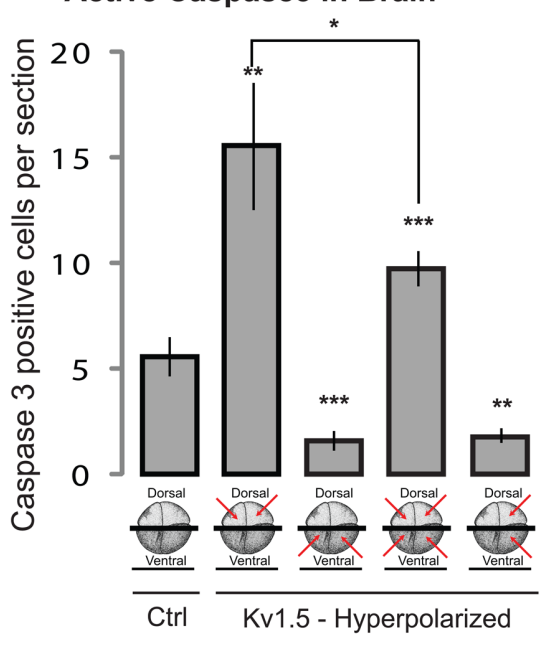

C

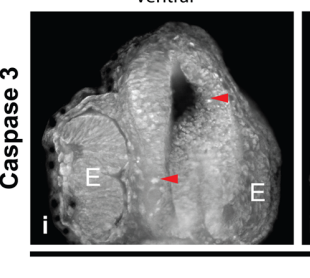

^ Black B-gal stain $\triangle$ Activated Caspase 3

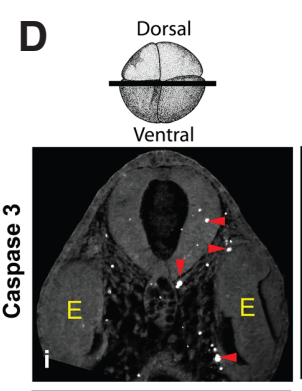

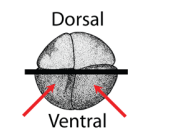
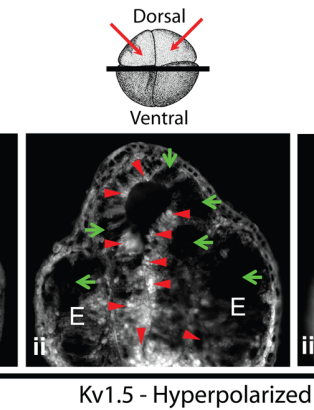

Ctrl

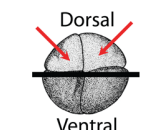

Ventral

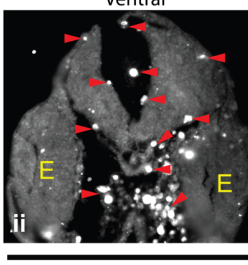

$\mathrm{Kv1} .5-$

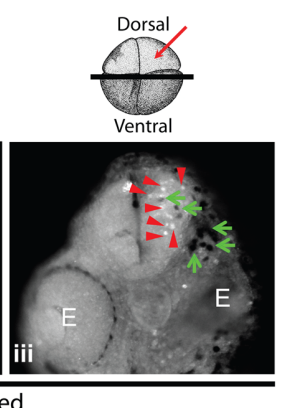

Eye

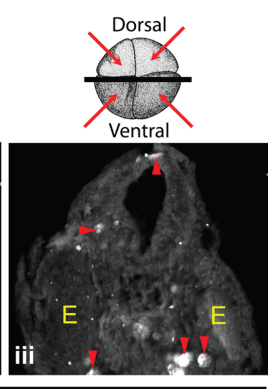

$\triangle$ Activated Caspase 3 E Eye
Fig. 2. Both local and distant $V_{\text {mem }}$ signals regulate apoptosis in the developing brain. (A) Illustration depicts the region of the cross-section shown. Agarose sections of stage 30 control (uninjected) embryos (i) and embryos microinjected with hyperpolarizing Kv1.5 mRNA (ii-v) in the indicated blastomeres (red arrows) at 4-cell stage. Immunostainng of sections through the developing brain with activated caspase 3 (i-v) shows a distinct change in the activated caspase 3 (red arrowheads) staining in the developing brain of microinjected embryos in comparison to uninjected controls. $E$ indicates eye tissue. (B) Quantification of activated caspase 3 immunostaining in the agarose sections through developing brains of stage 30 control (uninjected) and hyperpolarizing Kv1.5 microinjected (red arrows indicate injected blastomeres at 4-cell stage) embryos. Dorsal blastomere injections significantly increase the activated caspase 3 signal whereas ventral injections significantly decrease the activated caspase 3 signal. Both dorsal and ventral injections significantly decrease the activated caspase 3 signal in comparison to dorsal only injections. Values are mean + s.e.m. (n>10 for each group). $*, p<0.05 ; * *, p<0.01 ; * * *, p<0.001$ One way ANOVA with post-test. (C) Agarose sections of stage 30 Xenopus embryos co-injected with hyperpolarizing Kv1.5 and $\beta$-Galactosidase lineage tracer mRNA (i-iii) in the indicated blastomeres (red arrows) at 4-cell stage. Green arrowheads show developed $\beta$-Galactosidase reaction product which is seen as black (injected) regions on the sections. Immunostaining of sections through the developing brain with activated caspase 3 (i-iii) shows a distinct change in the activated caspase 3 (red arrowheads) staining in the developing brain of different microinjected embryos. Dorsal injected embryos show an increase in activated caspase 3 within the developing brain in comparison to ventrally

injected embryos. Left dorsal blastomere injection shows an increase in activated caspase 3 only on the injected side in comparison to the contralateral uninjected side. (D) Paraffin embedded thin sections of stage 30 control (uninjected) embryos (i) and embryos microinjected with hyperpolarizing Kv1.5 channel mRNA (ii,iii) in the indicated blastomeres (red arrows) at 4-cell stage.. Immunostainng of sections through the developing brain with activated caspase 3 (i-iii) shows a distinct increase in the activated caspase 3 (red arrowheads) staining in the developing brain and the region around the notochord of dorsally microinjected embryos (ii) in comparison to uninjected controls (i). Both dorsal and ventral injections (iii) shows a decrease in the activated caspase 3 staining in the developing brain and surrounding notochord region in comparison to the dorsally injected embryos (ii). 
(for targeting neural tissues) showed a highly significant increase in active caspase 3 signal within the embryonic brain (Fig. 2Aiii,B; $n>11$ embryos for each experimental group, one-way ANOVA, $p<0.001$, with post-tests). In addition to brain, elevated active caspase 3 signal was also seen in the regions immediately surrounding the brain region (Fig. 2Aiii). We verified our targeting of dorsal tissue by co-injection of $\beta$-galactosidase mRNA along with $K v 1.5$ and then performing the active caspase 3 immunostaining on sections of $\beta$-galactosidase-stained embryos (Fig. 2Ci-ii).

To get a better understanding of which specific tissues around the brain were exhibiting upregulation of apoptosis, we obtained thin sections through the brain after the embryos were embedded in paraffin and immunostained for active caspase 3 (Fig. 2D). In comparison to uninjected controls (Fig. 2Di) the active caspase 3 staining in dorsally $K v 1.5$-injected embryos was increased in the brain (as expected), but also in the region around the notochord, and in the developing eye (Fig. 2Dii). Injecting Kv1.5 (hyperpolarizing) mRNA in only one (left) dorsal cell of the embryo increases active caspase 3 signal only on the left brain of the embryo with the contralateral right side of the brain unchanged (Fig. 2Aii and Ciii), suggesting a local mode of action of dorsal $\mathrm{V}_{\text {mem }}$ signal in inducing apoptosis within the brain. Surprisingly, Kv1.5 (hyperpolarizing) mRNA injections in the ventral two blastomeres resulted in a significant decrease in the active caspase 3 signal in the developing brain in comparison to the controls (Fig. 2Aiv and B). Remarkably, injecting Kv1.5 (hyperpolarizing) mRNA in the both dorsal and ventral blastomeres showed significantly decreased active caspase 3 signal in comparison to the dorsal blastomeres injected embryos (Fig. 2A, B and D).

These results suggest that the dorsal $\mathrm{V}_{\text {mem }}$ pattern acts locally in regulating the extent and pattern of apoptosis in developing brain tissue. The ventral $V_{\text {mem }}$ pattern also regulates apoptosis in the developing brain tissue over long distance. Crucially, the dorsal and ventral $\mathrm{V}_{\text {mem }}$ patterns act counter to each other, in regulating the caspase 3 signal within the developing brain: remote hyperpolarization is sufficient to rescue local induction of apoptosis.

\section{Morphological brain defects induced by $V_{\text {mem }}$ perturbation are largely due to $V_{\text {mem }}$ regulation of apoptosis}

We next asked: to what extent are the brain morphological defects observed upon perturbing the dorsal $\mathrm{V}_{\text {mem }}$ patterns explained by the voltage-dependent apoptosis? We injected Kv1.5(hyperpolarizing) mRNA into two dorsal cells of four-cell embryos to disrupt normal brain development as before [Fig. 1 (Pai et al., 2015, Pai et al., 2012a)]. The chemical apoptosis inhibitor [M50054; (Tsuda et al., 2001)] was then used, from stage 10-30 (corresponding to neural tissue development), to block apoptosis in a suppression analysis strategy. The concentration of the apoptosis inhibitor used was that which did not produce any brain morphological defects by itself (Fig. 3A). The embryos were allowed to develop until stage 45 and scored for brain morphology defects as previously documented (Fig. 1). Dorsal blastomere injections of $\sim 2.2-3 \mathrm{ng} K v 1.5 \mathrm{mRNA}$ resulted in significant increase in the incidence of misformed brain in comparison to uninjected controls as expected (Fig. 3B). Treating Kv1.5 mRNA-injected embryos with M50054 (20 $\mu \mathrm{M})$ resulted in near complete prevention of the effect of $K v 1.5$ mRNA injections (Fig. 3B; $\chi^{2}$ test, $p<0.001$, post test ${ }^{* *} p<0.01$ ). We conclude that $\mathrm{V}_{\text {mem }}$ regulation of apoptosis within the developing brain tissue is a major contributor to the induction of brain morphology defects resulting from perturbation of $\mathrm{V}_{\text {mem }}$ patterning, since such defects can be largely prevented by inhibiting apoptosis.

\section{Both local and distant $V_{\text {mem }}$ signals regulate proliferation in the developing brain}

In addition to apoptosis, proliferation is a major factor regulating tissue boundaries and organ size during development and regeneration (Chan et al., 2002, Joseph and Hermanson, 2010, Shitamukai and Matsuzaki, 2012, Stanger, 2008a, Stanger, 2008b). Our previous study showed that $\mathrm{V}_{\text {mem }}$ affects proliferation in the developing brain (Pai et al., 2015), as it does in a number of normal and neoplastic tissues (Blackiston et al., 2011, Blackiston et al., 2009, Chernet and Levin, 2013a, Chernet and Levin, 2014, Ding et al., 2012, Higashimori and Sontheimer, 2007, Nilius and Wohlrab, 1992, Yang and Brackenbury, 2013, Zhang et al., 2012). Hence, we next analyzed proliferation within the developing brain of Kv1.5 (hyperpolarizing) mRNA injected Xenopus embryos by immunostaining transverse sections through brain, for phosphorylated histone 3B (H3P; a proliferation marker; (Saka and Smith, 2001, Sanchez Alvarado, 2003). Sections anterior to, or through, the eye were used for analysis of developing brain tissue. Control

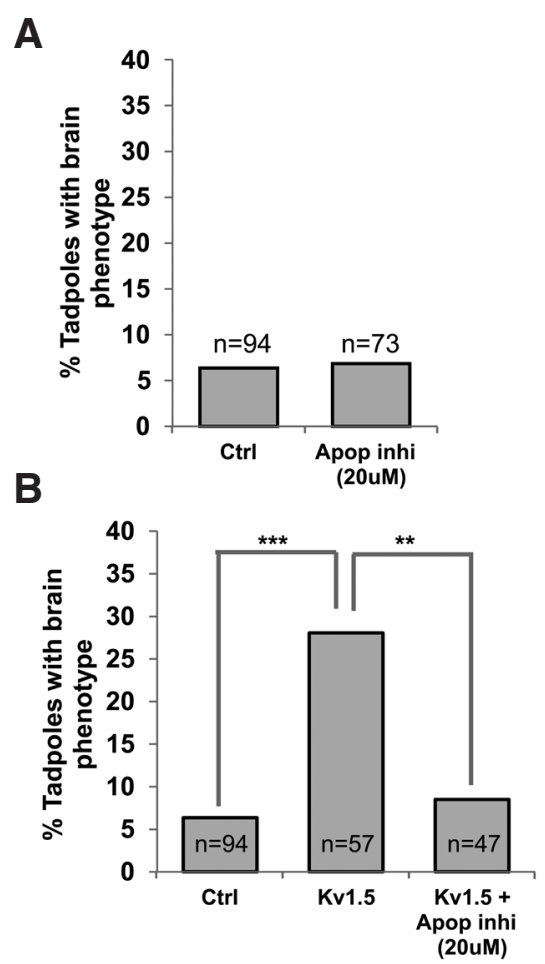

Fig. 3. Inhibition of apoptosis rescues the Kv1.5 induced brain mispatterning. (A) Quantification of tadpoles with brain phenotypes in control (untreated) and apoptosis inhibitor treated (M50054 - 20uM) from stage $10-30$ of Xenopus embryos. No significant change was seen on treatment of embryos with the apoptosis inhibitors in comparison to controls. T-test analysis was performed. (B) Quantification of tadpoles with malformed brain phenotypes in control (uninjected) and hyperpolarizing Kv1.5 microinjected (dorsal 2 blastomere at 4-cell stage) embryos with or without the apoptosis inhibitor (M50054-20uM; treated from stage 10-30). A $\chi^{2}$ analysis showed significant variance among the groups. A significantly high incidence of malformed brain phenotype is seen in Kv1.5 microinjected embryos (post t-test $\left.{ }^{* *}, p<0.001\right)$. This effect of Kv1.5 is significantly prevented by Apoptosis inhibitor (M50054 - 20uM) (post t-test, $\left.{ }^{* *}{ }^{*}, p<0.001\right)$. 
(uninjected) embryos show H3P signal mainly adjacent to the neural canal (Fig. 4Ai). This observation is consistent with previous reports identifying this region as a niche for pluripotent cells from which neural progenitor cells are generated (Chan et al., 2002, Gotz and Huttner, 2005, Stanger, 2008a, Stanger, 2008b). Embryos injected with Kv1.5 (hyperpolarizing) mRNA into the dorsal two cells at four-cell stage (for targeting neural tissues) significantly decreased the H3P signal in the embryonic brain in comparison to the controls (Fig. 4Aiii, $B ; n>11$ embryos for each experimental group, one-way ANOVA, $p<0.001$, with post-tests). We verified our targeting of dorsal tissue by co-injection of $\beta$-galactosidase mRNA along with Kv1.5 (hyperpolarizing) mRNA and then performing the H3P immunostaining on sections of $\beta$-galactosidase-stained embryos (Fig. 4Cii). Injecting Kv1.5 (hyperpolarizing) mRNA in only one dorsal blastomere slightly (non-significantly) decreased H3P signal in the developing brain in comparison to controls (Fig. 4Aii). Interestingly, Kv1.5 (hyperpolarizing) mRNA injections in the ventral two blastomeres at four-cell stage, significantly increased
$\mathrm{H} 3 \mathrm{P}$ staining in the developing brain and eye in comparison to controls (Fig. 4Aiv, B and $\mathrm{Ci}$ ), confirming the long distance action of ventral $V_{\text {mem }}$ patterns in upregulating proliferation in the developing brain, and once again ruling out nonspecific toxicity as an explanation of the consequences channel-induced voltage change. Dorsal injections decreased the proliferation, consistent with the known hyperpolarized state of quiescent (non-proliferative) cells (Binggeli and Weinstein, 1986a, Blackiston et al., 2009). Remarkably, Kv1.5 (hyperpolarizing) mRNA injections into both dorsal and ventral blastomeres were not intermediate between the high ventral and low dorsal outcomes, but were even higher than ventral alone. Injection into both dorsal and ventral regions induced significantly increased H3P signal in the developing brain and eye (Fig. 4Av and B) suggesting a predominant effect of the ventral $\mathrm{V}_{\text {mem }}$ patterns on H3P signal within the developing brain.

The most striking was the effect of one-sided injections (left two blastomeres at four-cell stage), after which H3P signal within the brain significantly increased on both the injected ( $\beta$-galactosidase
A
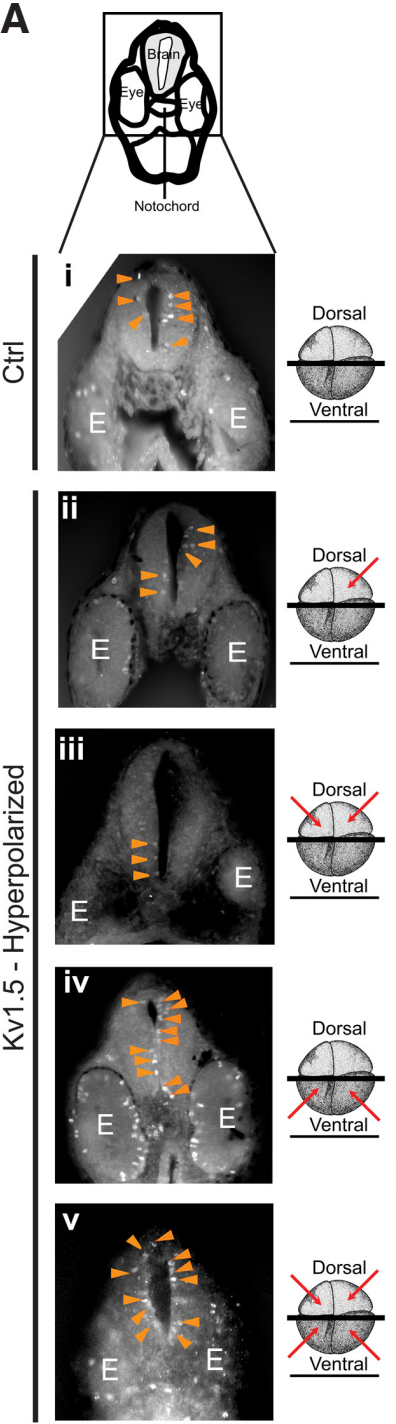

B
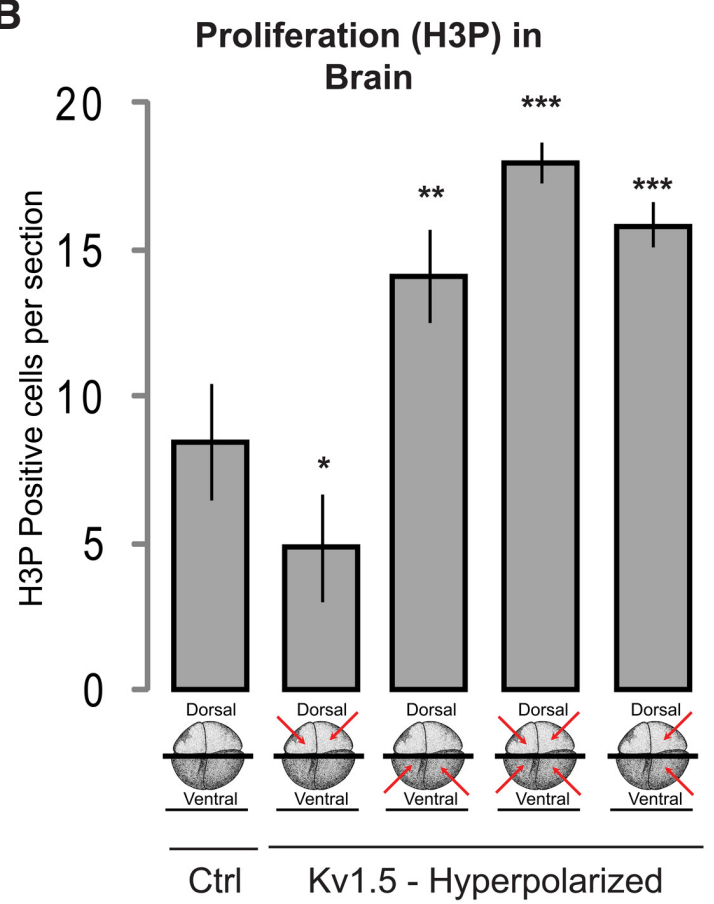

C
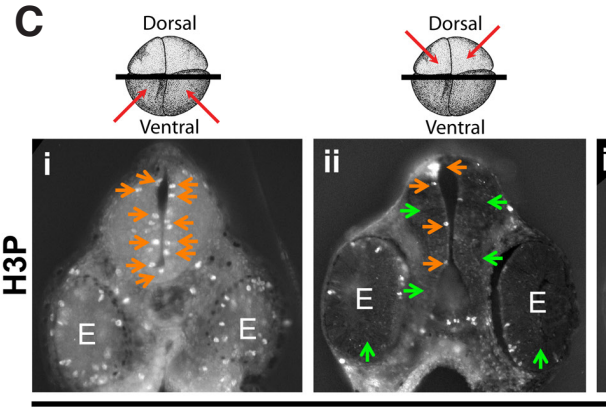

Kv1.5 - Hyperpolarized

$\uparrow$ Black B-gal stain $\uparrow \mathrm{H} 3 \mathrm{P}$ E Eye
Fig. 4. Both local and distant $V_{\text {mem }}$ signals regulate proliferation in the developing brain. (A) Illustration depicts the region of cross-section shown. Agarose sections of stage 30 control (uninjected) embryos (i) and embryos microinjected with hyperpolarizing Kv1.5 mRNA (ii-v) in the indicated blastomeres (red arrows) at 4-cell stage. Immunostainng of sections through the developing brain with H3P (i-v) shows a distinct change in the H3P (orange arrowheads) staining in the developing brain of microinjected embryos in comparison to uninjected controls. (B) Quantification of $\mathrm{H} 3 \mathrm{P}$ immunostaining in the agarose sections through developing brains of stage 30 control (uninjected) and hyperpolarizing Kv1.5 microinjected (red arrows indicate injected blastomeres at 4-cell stage) embryos. Dorsal blastomere injections significantly decrease the H3P signal whereas ventral injections significantly increase the H3P signal. Injections of all 4 blastomeres significantly increases the H3P signal in comparison to dorsal only injections. Values are mean + s.e.m. ( $n>8$ for each group). $*, p<0.05 ; * *, p<0.01 ; * * *, p<0.001$ One way ANOVA with post-test. (C) Agarose sections of stage 30 Xenopus embryos co-injected with hyperpolarizing Kv1.5 and $\beta$-Galactosidase lineage tracer mRNA (i-iii) in the indicated blastomeres (red arrows) at 4-cell stage. Green arrowheads show developed $\beta$-Galactosidase stain which is seen as black (injected) regions on the sections. Immunostaining of sections through the developing brain with H3P (i-iii) shows a distinct change in the H3P (orange arrows) staining in the developing brain of different

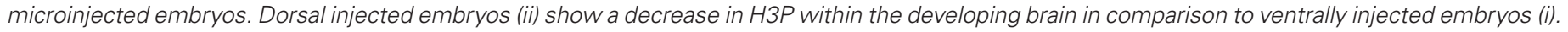

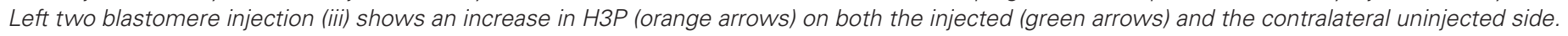



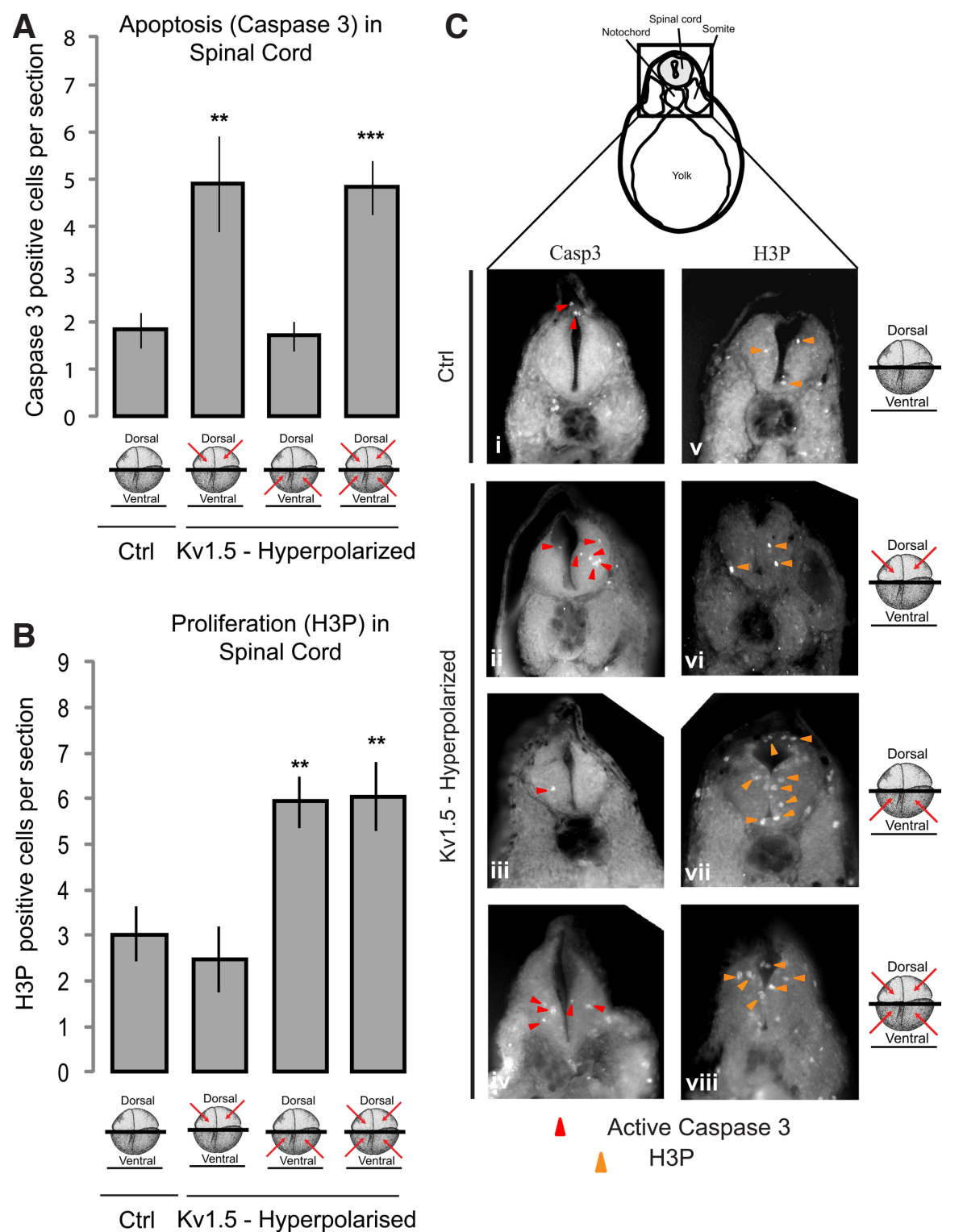

Fig. 5. Both local and distant $V_{\text {mem }}$ signals regulate apoptosis and proliferation in the developing spinal cord. (A) Quantification of activated caspase 3 immunostaining in the agarose sections through developing spinal cord of stage 30 control (uninjected) and hyperpolarizing Kv1.5 microinjected (red arrows indicate injected blastomeres at 4-cell stage) embryos. Dorsal blastomere injections significantly increase the activated caspase 3 signal whereas ventral injections show no change in activated caspase 3 signal. Both dorsal and ventral injections significantly increase the activated caspase 3 signal, similar to dorsal only injections, in comparison to controls. Values are mean + s.e.m. $\left(n>10\right.$ for each group). ${ }^{*}, p<0.01 ; * * *, p<0.001$ One way ANOVA with posttest. (B) Quantification of H3P immunostaining in the agarose sections through developing spinal cord of stage 30 control (uninjected) and hyperpolarizing Kv1.5 microinjected (red arrows indicate injected blastomeres at 4-cell stage) embryos. Dorsal blastomere injections slightly decrease the H3P signal whereas ventral injections significantly increase the H3P signal. Both dorsal and ventral injections significantly increase the H3P signal in comparison to dorsal only injections. Values are mean + s.e.m. ( $n>8$ for each group). **, $p<0.01$ One way ANOVA with post-test. (C) Illustration depicts the region of the cross-section shown. Agarose sections of stage 30 control (uninjected) embryos ( $i$ and $v$ ) and embryos microinjected with hyperpolarizing Kv1.5 mRNA (ii-iv and vi-viii) in the indicated blastomeres (red arrows) at 4-cell stage. Immunostaining of sections through the developing spinal cord with activated caspase 3 (ii-iv) shows a distinct change in the activated caspase 3 (red arrowheads) staining in the developing spinal cord of microinjected embryos in comparison to uninjected controls and ventral injections. Immunostaining of sections through the developing spinal cord with H3P (vi-viii) shows a distinct change in the activated caspase 3 (orange arrowheads) staining in the developing spinal cord of microinjected embryos in comparison to uninjected controls and dorsal injections. positive) and the contralateral uninjected $(\beta-$ galactosidase negative) side, revealing that ventral $\mathrm{V}_{\text {mem }}$ state signaling crosses the midline in regulating proliferation (Fig. $4 \mathrm{Ciii}$ ). These results suggest that dorsal $\mathrm{V}_{\text {mem }}$ patterns act locally, reducing proliferation with the developing brain tissue. Contrarily, the ventral $\mathrm{V}_{\text {mem }}$ patterns act at a distance, enhancing the proliferation within the developing brain tissue. The results suggest that the ventral $V_{\text {mem }}$ pattern's effect on proliferation within the developing brain tissue is dominant, and underline the long-range nature of bioelectric signaling which crosses both the dorso-ventral and also the left-right axes.

Spinal cord apoptosis is regulated only by local $V_{\text {mem }}$ signal and proliferation only by long-distance $V_{\text {mem }}$ signal

Do $V_{\text {mem }}$ patterns play a similar role in other aspects of CNS, particularly spinal cord development? To assess this, we analyzed apoptosis and proliferation (as described in detail above) within the developing spinal cord of $\mathrm{V}_{\text {mem }}$-perturbed (hyperpolarized by Kv1.5 mRNA injections) Xenopus embryos by immunostaining the transverse sections through the developing spinal cord, for activated caspase 3 and $\mathrm{H} 3 \mathrm{P}$ respectively. Sections posterior to the otic/ear vesicles and through the yolk were used for analysis of developing spinal cord. In control (uninjected) embryos active caspase 3 was intermittently and sparsely distributed within the nerve cord similar to brain tissue (Fig. 5A and Ci). Embryos injected with Kv1.5 (hyperpolarizing) mRNA into the dorsal two cells at four-cell stage (for targeting neural tissues) showed significantly increased active caspase 3 signal within the embryonic spinal cord in comparison to controls (Fig. 5A and Cii; $n>10$ for each experimental group, one-way ANOVA, $p<0.001$, with post-tests). Contrary to the observations in the brain tissue, Kv1.5(hyperpolarizing) mRNA injections in the ventral two blastomeres had no effect on active caspase 3 signal (Fig. 5A and Ciii). Analogously, Kv1.5 (hyperpolarizing) mRNA injections in both the dorsal and ventral blastomeres showed no change in the active caspase 3 signal in comparison to the dorsal blastomere-injected embryos, which exhibited the expected increase in apoptosis (Fig. 5Aand Civ). These results suggest that although the local (dorsal) $V_{\text {mem }}$ patterns regulate apoptosis in the developing spinal cord similar to that in the developing brain, the ventral $\mathrm{V}_{\text {mem }}$ pattern has no bearing on regulation of apoptosis in 
the developing spinal cord unlike within the developing brain.

When assessed for proliferation, in control (uninjected) embryos the H3P signal was mainly present adjacent to the neural canal of the developing spinal cord, similar to the developing brain pattern (Fig. 5B and Cv). Kv1.5 (hyperpolarizing) mRNA injection into the dorsal two cells at four-cell stage (for targeting neural tissue) showed no change in the H3P signal within the developing spinal cord in comparison to controls (Fig. 5B and Cvi; $n>8$ for each experimental group, one-way ANOVA, $p<0.001$, with post-tests). Kv1.5 (hyperpolarizing) mRNA injections into the ventral two blastomeres (targeting non-neural tissue) significantly increased $\mathrm{H} 3 \mathrm{P}$ signal in the developing spinal cord in comparison to controls, similar to the effect seen in the developing brain (Fig. 5B and Cvii). Kv1.5 (hyperpolarizing) injections in both the dorsal and ventral blastomeres at four-cell stage had unchanged levels of H3P signal in comparison to the dorsal two blastomeres injected embryos (Fig. 5B and Cviii). These results suggest that although the long distance (ventral) $V_{\text {mem }}$ patterns regulate proliferation within the developing spinal cord similar to the developing brain, the dorsal (local) $\mathrm{V}_{\text {mem }}$ pattern has no bearing on regulation of proliferation in the developing spinal cord unlike the developing brain.

These data reveal a simpler regulation of apoptosis (by local dorsal $\mathrm{V}_{\text {mem }}$ patterns) and proliferation (by distant ventral $\mathrm{V}_{\text {mem }}$ pattern) within the developing spinal cord in comparison to the developing brain. We conclude that the anterior and posterior aspects of the CNS both utilize bioelectric signaling but in distinct spatial modes.

\section{Discussion}

\section{Membrane voltage potential signals over long-distance im- pinge upon developmental brain morphology}

Dynamic changes in resting membrane potentials $\left(\mathrm{V}_{\mathrm{mem}}\right)$ carry patterning information during embryonic organ development in Xenopus (Adams and Levin, 2013, Levin, 2014a, Mustard and Levin, 2014, Tseng and Levin, 2013b, Vandenberg et al., 2011). In addition to activity-dependent sculpting of neural connections (Kozorovitskiy et al., 2012, Penn and Shatz, 1999), the spatial distribution patterns of $\mathrm{V}_{\text {mem }}$ in developing embryos controls aspects of large-scale morphogenesis of the nervous system, particularly eye and brain (Beane et al., 2013, Pai et al., 2015, Pai et al., 2012a, Pai et al., 2012b). Here we assess the effect of $\mathrm{V}_{\text {mem }}$ specifically on apoptosis and proliferation within the developing central nervous system (CNS) as it regulates the large-scale morphogenesis of the brain and spinal cord. Particularly we examine the effect of local and long-distance $\mathrm{V}_{\text {mem }}$ signals in regulating these cell processes.

Molecularly and spatially regulating $\mathrm{V}_{\text {mem }}$ with microinjections of well-characterized ion channel mRNAs is a very tractable and well established experimental method (Adams and Levin, 2013). It erases the endogenous differential spatial distribution patterns of $\mathrm{V}_{\text {mem }}$ of neural and non-neural tissues that encode patterning information necessary proper brain morphogenesis (Pai et al., 2015). While here we focused our analysis on the most potent hyperpolarizer, Kv1.5, our previous work showed the effect is truly voltage-dependent, and not tied to a specific ion channel protein, as many other ion translocators with the same effect on $\mathrm{V}_{\text {mem }}$ can be substituted (Pai et al., 2015, Pai et al., 2012a).

Although absolute $V_{\text {mem }}$ is known to control cell proliferation and differentiation (Blackiston et al., 2009, Sundelacruz et al., 2008, Sundelacruz et al., 2009, Sundelacruz et al., 2013), the effect of relative $\mathrm{V}_{\text {mem }}$ differences between groups of cells on cellular and tissue processes is only beginning to be understood. Cell autonomous local bioelectric signaling has been observed in eye (Pai et al., 2012a) and brain (Pai et al., 2015) patterning, and is mainly transduced by intracellular calcium signaling and GJCs to regulate cellular behavior. Non-cell-autonomous bioelectric signaling has been observed in brain development (Pai et al., 2015), tumor suppression (Chernet et al., 2014), ectopic innervation (Blackiston et al., 2015a), and left-right patterning (Levin and Mercola, 1999). While the transduction mechanisms of such effects are beginning to be understood, the extent of such signaling during normal development has not been explored. Understanding this interplay between these local and long-distance bioelectric transduction mechanism is critical in understanding topology of signaling resulting in neural development.

This study attempts to tease apart contributions of relative $\mathrm{V}_{\text {mem }}$ levels during normal development of CNS. Altering or erasing the characteristic local hyperpolarization pattern within the developing neural tube leads to defects in brain patterning ranging from small or absent nostrils and forebrain, deformed or shrunken midbrain and deformed eyes, while the rest of the animal developed normally [Fig. 1 and (Pai et al., 2015, Pai et al., 2012a)]. Hence the local $V_{\text {mem }}$ pattern seems to function as a distinct bioelectric signal impinging on brain patterning (Pai et al., 2015, Pai et al., 2012a). However, a near complete suppression or rescue of this phenotype is achieved by hyperpolarizing the distant (ventral) region (which directly does not contribute to neural development) (Fig. 1), suggesting distant $\mathrm{V}_{\text {mem }}$ patterns from surrounding tissues also potentially impinge on brain patterning. These results suggests that during embryonic development, at least in brain patterning, information from both local and distant bioelectric patterns is read and incorporated towards correct morphological patterning of the brain tissue.

\section{Local and distant $V_{\text {mem }}$ patterns regulate apoptosis and pro- liferation within the developing brain \\ Disrupting or erasing the local (dorsal, neural) endogenous} $V_{\text {mem }}$ gradient pattern within the developing neural tube increased apoptosis (Fig. 2) and decreased proliferation (Fig. 4) within the developing brain and eye. Inhibiting apoptosis with chemical inhibitors reduced the ability of $\mathrm{V}_{\text {mem }}$ disruption to cause brain mispatterning (Fig. 3). These results suggest that local (dorsal, neural) bioelectric signals' regulation of apoptosis is an important endogenous component of proper brain tissue morphology during development (Fig. 1); this effect is in addition to voltage regulation of proliferation. Perturbing distant (ventral, non-neural) bioelectric patterns decreased apoptosis (Fig. 2) and strongly increased proliferation (Fig. 4) within the developing brain. Perturbing both distant (ventral, non-neural) and local bioelectric states resulted in reduced apoptosis in comparison to perturbation of local bioelectric states alone (Fig. 2). In case of proliferation, the distant (ventral, non-neural) bioelectric cell states were able to completely override the effect of local bioelectric signal perturbation (Fig. 4). These results suggest that both local (dorsal, neural) and distant (ventral, non-neural) bioelectric signals regulate cellular behavior (apoptosis and proliferation) within the developing brain, in opposite directions. In case of apoptosis, since the effect of perturbing distant bioelectric signals only partially reverse the effect of local bioelectric signal (Fig. 2), it can be postulated that the local (dorsal, neural) bioelectric signals are the dominant of the two. 
Contrarily, in case of proliferation, since effect of perturbing the distant bioelectric signal overrides the effect of local bioelectric signals on proliferation, it suggests the distant (ventral, non-neural) bioelectric signals are the dominant of the two.

Although perturbing local (dorsal, neural) bioelectric signals increases apoptosis in the developing brain, it cannot be attributed to toxicity or merely disruption of housekeeping processes since perturbing both local and distant (ventral, non-neural) bioelectric signal actually decreases apoptosis in the brain. Instead it suggests instructive signaling by bioelectric signals as shown for bioelectric signals in other contexts (Konig et al., 2004, Ng et al., 2010, Pai et al., 2015, Pai et al., 2012a, Pai et al., 2012b, Sundelacruz et al., 2009, van Vliet et al., 2010). Further, it suggests integration of information from more than one bioelectric signal (local and distant) converging towards regulation of particular cell/tissue process during embryonic organ patterning.

Apart from developing brain, bioelectric signal also regulate apoptosis in region around the developing brain particularly in cells surrounding the notochord (not within the notochord) (Fig. 2). Notochord and notochord-derived signals are absolutely crucial in induction of the neural plate, formation of neural tube and differentiation of regions of neural tube (Altmann and Brivanlou, 2001). During embryonic development such inductive interactions between germ layers and between tissues are critical in achieving target morphologies of organs (Tannahill et al., 2005). Apoptosis in these cells around the notochord and the developing brain could disrupt such critical inductive interactions by either eliminating signal producing cells or signal receiving cells. Such secondary effect of apoptosis on inductive interactions during brain development have previously been documented (Nonomura et al., 2013). Hence, this apoptosis in the regions around notochord and developing brain may also be responsible for the observed defects in brain morphology upon perturbation of bioelectric signals. The specific nature of the inductive interactions that may be disrupted by this bioelectric signal-regulated apoptosis and how they affect embryonic brain morphology and patterning remains an area of active investigation.

An interesting observation in bioelectric regulation of apoptosis within the developing brain is that the distant (ventral, non-neural) bioelectric signals regulate apoptosis in the developing brain across the dorso-ventral axis, however, the local (dorsal, neural) bioelectric signal is highly localized and does not act across the midline from the left to right side of the developing neural tissue (Fig. 2). Unlike the predominant (local) bioelectric regulation of apoptosis which does not cross the midline and is highly local, the predominant (distant) bioelectric regulation of proliferation functions across the dorso-ventral as well as the midline (left-right) axis (Fig. 4). How is such tight spatial control achieved between the local and distant bioelectric signals? And more important what is the evolutionary significance or advantage of such spatially differential control of apoptosis and proliferation by bioelectrical signals in generating the target morphology of the embryonic brain? These aspects remain to be probed in subsequent studies.

Together, these observations reveal the important interplay between the local and distant bioelectric states in regulating apoptosis and proliferation within the developing embryonic brain. Patterning information from both types of bioelectric signals is integrated towards generating proper brain morphology in the developing embryo.

\section{Local and distant $V_{\text {mem }}$ patterns have counterbalancing effects on apoptosis and proliferation within the developing brain}

Both apoptosis and proliferation within the developing brain have at least two controls: local bioelectric signal and distant bioelectric signal. Interestingly, these two bioelectric controls have opposite actions (one decreases, other increases) on the same process (proliferation or apoptosis) (Fig. 2 and 4). Such coupling of two bioelectric counter controls converging on one cellular process (in this case proliferation or apoptosis) result in a system that is able to finely tune the extent of that cellular process to meet the morphological and physiological requirements of the developing embryo. Interestingly, such coupling of opposite processes is seen at a higher level of complexity with the local bioelectric signals being predominant in regulating apoptosis whereas the distant bioelectric signals being predominant in regulating proliferation within the same tissue (developing brain). Such dueling controls could be important in regulating the size of a tissues/organs in relation to the size of embryo/organism as a whole, by coupling the dynamic morphology of growing structures to the physiological state of surrounding (or even distant) tissues in vivo.

\section{Bioelectric signal regulate apoptosis and proliferation within the developing brain}

What could be the mechanism employed by the bioelectrical signals towards regulation of apoptosis and proliferation in the embryonic brain? There are several possibilities, currently under investigation.

It is possible that perturbing the distant bioelectric signals results in mispatterning of ventral components of the neural structures (such as notochord) that play a role in inductive interactions resulting in formation of the neural plate, neural tube and the brain (Altmann and Brivanlou, 2001, Tannahill et al., 2005). However, this hypothesis is less likely, as in such a situation at least some incidence of mispatterned ventral tissues would occur, which we do not see in our experimental groups (notochord, gut and endodermal structures all form normally). Another possible mechanism of action of distant bioelectric signals could be through regulation of morphogen production/response in the inductive tissues like the notochord (Tannahill et al., 2005). Notochord releases morphogens like serotonin (5-HT) and sonic hedgehog (Shh) which regulate apoptosis, proliferation, and differentiation within the embryonic developing brain (Lauder et al., 1981, Tannahill et al., 2005). Bioelectric signal-mediated regulation of morphogen synthesis and signaling has been observed at the transcriptional level in Xenopus embryos (Pai et al., in review). Thus notochord and its morphogens, may act as a sensor or integrator system of the bioelectric signals (both local and distant) towards appropriate embryonic brain patterning. Interestingly, notochord is formed before neural tissue specification and is located at the nexus of dorsal-ventral and left-right body axis, an ideal location for a sensor/integrator.

Previous studies have shown a critical role of gap junction channels (GJCs) in transducing the effect of distant bioelectric signal perturbations on proliferation within the developing brain (Pai et al., 2015). Movement of serotonin through GJCs has also been shown to be an important component of distant bioelectric communication guiding ectopic nerve growth (Blackiston et al., 2015a). Finally, modeling and experimental testing of model predictions has revealed that the left and right sides of the Xenopus embryo produce a coherent long-range bioelectric growth-control 
signal that is coordinated by the action of GJCs (Chernet et al., 2014). Disrupting GJC communication on one side of the embryo affected $\mathrm{V}_{\text {mem }}$-driven physiological changes (rate of tumor formation in oncogene-injected embryos) on the other side (Chernet et al., 2014). A similar GJCs-mediated mechanism could also be responsible for transducing the distant bioelectric signals regulating apoptosis within the developing brain. Alternatively, GJCs could serve as conduits for transducing bioelectric patterning information to sensor/integrators (like notochord as discussed above) tissues which in turn regulate the levels of apoptosis and proliferation in the developing brain. Cell to cell gap junction connections are quite dynamic during embryonic development (Anava et al., 2013, Warner, 1985), and the sensitivity of GJs to transjunctional voltage (Palacios-Prado and Bukauskas, 2009, Verselis et al., 1991) suggest gap junctions as the ideal candidate to mediate cells' ability to compare their voltage with neighboring cell groups. The molecular identity of the signal (ionic, biochemical, or both) passing through GJCs communicating this information is not yet known, although prior work suggests neurotransmitters such as serotonin as a likely candidate for subsequent analysis (Blackiston et al., 2011, Blackiston et al., 2015b, Fukumoto et al., 2005, Lobikin et al., 2012a). Understanding the precise transduction mechanism of local and
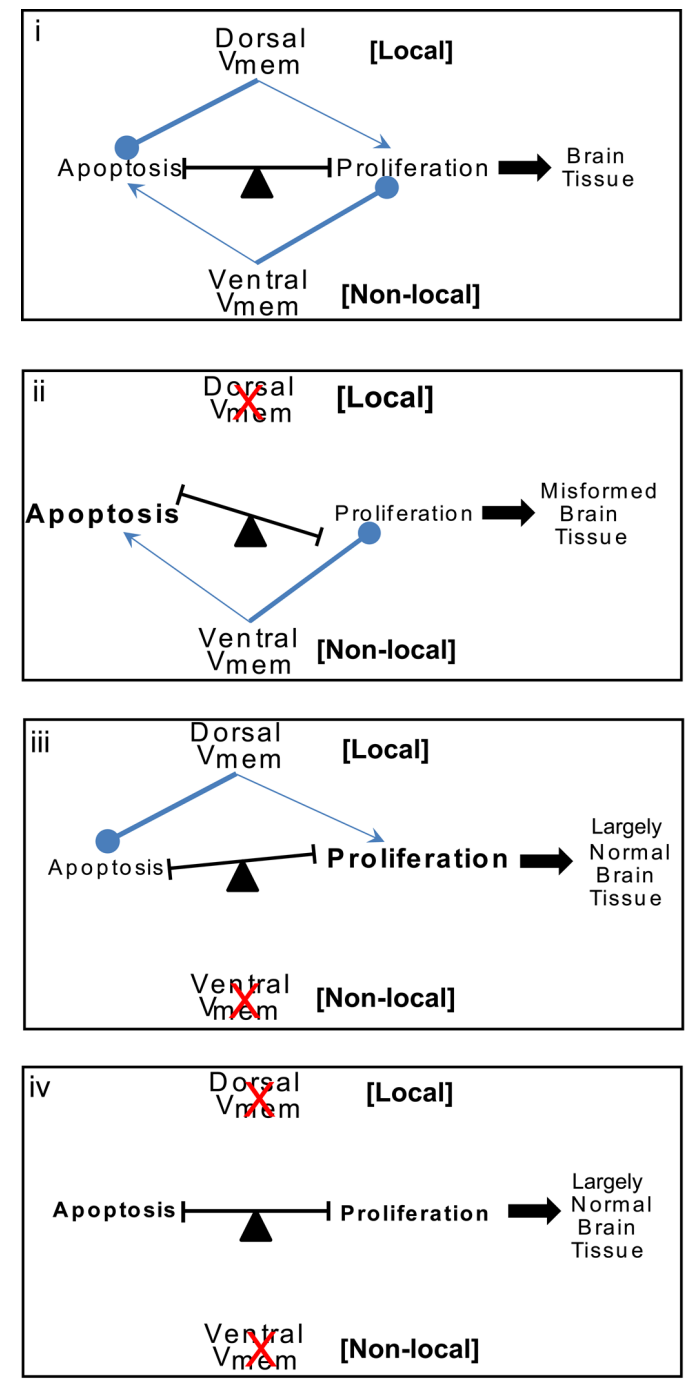

distant bioelectric signals in regulating brain patterning remains an active area of investigation.

\section{Bioelectric signal regulate apoptosis and proliferation in em- bryonic spinal cord development}

The bioelectric regulation of apoptosis and proliferation in the developing spinal cord appears to be simpler with less regulation than in the developing brain. Apoptosis in the developing spinal cord is regulated only by the local bioelectric signal with no detectable input from distant bioelectric signals on apoptosis (Fig. 5). In contrast, proliferation in the developing spinal cord is regulated only by the distant bioelectric signal with no detectable input from the local bioelectric signals on proliferation at the relevant developmental period (Fig. 5). Moreover, in comparison to brain development, the dominant bioelectric signals (local for apoptosis and distant for proliferation) seem to persist but the subtler/weaker bioelectric signals (distant for apoptosis and local for proliferation) seem to be absent.

Why is the bioelectric signal regulation of apoptosis and proliferation in embryonic spinal cord development simplified in comparison to the developing brain is not yet known. The brain has been postulated to have evolved from the cephalization (bulging or growing) of the anterior end of the neural tube/spinal cord of cephalochordates like Amphioxus (Northcutt, 1996, Northcutt, 2003). Could the subtle bioelectric controls seen in brain development but absent in spinal cord development be a part of brain evolutionary mechanism from rudimentary neural tube/ spinal cord? This hypothesis will be addressed in future work.

\section{A model integrating the bioelectrical signals in regulating apoptosis and proliferation in brain development}

We suggest a model integrating the collective data on bioelectric signals controlling apoptosis and proliferation during brain patterning from this and previous study (Fig. 6). The instructive bioelectric signal $\left(\mathrm{V}_{\text {mem }}\right.$ state $)$ is physiological in nature and hence not identical with any one gene product (ion channel), as numerous ion translocators contribute to overall $\mathrm{V}_{\text {mem }}$ and can compensate for one another in setting correct voltage state during pattern formation (Blackiston et al., 2011, Pai et al., 2012a). This aspect is extremely desirable from the perspective of developing therapeutic applications as it enables a broad range of potential reagents and endogenous targets for

Fig. 6. A model for $V_{\text {mem }}$ regulation of apoptosis-proliferation balance towards shaping brain morphology. Model for $V_{\text {mem }}$ regulation of brain tissue sculpting. Both dorsal and ventral $V_{\text {mem }}$ signals are involved in brain tissue sculpting by their effects on the balance of apoptosis and proliferation within the tissue. Undernormal conditions (i) the dorsal $V_{\text {mem }}$ strongly inhibits apoptosis and mildly stimulates proliferation locally within the developing brain. The ventral $V_{\text {mem }}$ strongly inhibits proliferation and mildly stimulates apoptosis at a distance in the developing brain tissue. Coordination of these two $V_{\text {mem }}$ signals results in proper balance of proliferation-apoptosis and ultimately sculpting of the brain tissue during development. Blocking of dorsal $V_{\text {mem }}$ signal (ii) releases the strong inhibition on apoptosis and further facilitates strong inhibition of proliferation by the ventral $V_{\text {mem }}$ signals. This results in a shift in balance towards apoptosis resulting in malformed brain tissue. Blocking of ventral $V_{\text {mem }}$ signal (iii) releases the strong inhibition on proliferation and increases the inhibition of apoptosis by the dorsal $V_{\text {mem }}$ signal. This results in significant increase in proliferating cells but otherwise largely normal brain structure development. Blocking of both dorsal and ventral $V_{\text {mem }}$ signal (iv) cancel each other's effect on proliferation and apoptosis leaving the sculpting of brain to other regulators of brain structure development resulting in largely normal brain structure. 
pharmacological treatment of brain malformation defects.

Disturbing the endogenous local (dorsal, neural) bioelectric pattern leads to increase in apoptosis and decrease in proliferation in the developing brain (Fig. 6ii). The ensuing shift in the apoptosisproliferation balance towards apoptosis leads to mispatterning of the developing brain (Fig. 6ii). Further, inhibiting apoptosis prevents this shift in balance towards apoptosis and rescues the embryos from the bioelectric mispatterning. Disturbing the endogenous distant (ventral, non-neural) bioelectric signal leads to decrease in apoptosis and increased proliferation with a largely normally patterned brain (Fig. 6iii). While a role of bioelectric signal in regulating apoptosis-proliferation has been shown in determining planarian regeneration (Beane et al., 2013), this is the first account of bioelectric signal (particularly contribution of both local and distant bioelectric signals) controlling the apoptosis-proliferation balance in sculpting the neural tissue in a developing vertebrate embryo.

\section{Conclusion}

We have shown that endogenous patterns of bioelectric signals are key determinants of CNS development. The bioelectric signals of local and distant tissues are important endogenous components involved in brain and spinal cord sculpting particularly at the level of regulating apoptosis and cell proliferation. We reveal a complex counter-balancing interaction of local and distant bioelectric cell states in regulating key morphology determining cellular behaviors of apoptosis and proliferation. Taken together, these data show a new function of bioelectric signals in controlling the apoptosisproliferation balance during embryonic brain and spinal cord patterning. Especially interesting are the long-range rescue effects, which suggest a clear roadmap using ion channel drugs (electroceuticals) targeted to non-neural tissues as a tractable strategy for manipulating neurogenesis and neural patterning in the context of birth defects, regenerative medicine, and synthetic bioengineering.

\section{Materials and Methods}

\section{Animal husbandry}

Xenopus laevis embryos were fertilized in vitro according to standard protocols (Sive et al., 2000) in 0.1X Marc's Modified Ringer's solution (MMR; $\left.10 \mathrm{mM} \mathrm{Na}^{+}, 0.2 \mathrm{mM} \mathrm{K}^{+}, 10.5 \mathrm{mM} \mathrm{Cl}^{+}, 0.2 \mathrm{mM} \mathrm{Ca}^{2+}, \mathrm{pH} 7.8\right)$. Xenopusembryos were housed at $14-18^{\circ} \mathrm{C}\left(14^{\circ} \mathrm{C}\right.$ overnight after injection and subsequently at $18^{\circ} \mathrm{C}$ ) and staged according to Nieuwkoop and Faber (Nieuwkoop and Faber, 1967). PNTub::GFP transgenic Xenopus were created as described (Kroll and Amaya, 1996) except that the restriction enzyme was omitted (Lin et al., 2012, Marsh-Armstrong et al., 1999). There is no practical way to precisely determine the sex of the embryos at the stages at which these procedures are done, and the ratio of male:female should be 50:50 in all of our experiments. All experiments were approved by the Tufts University Animal Research Committee (M2014-79) in accordance with the guide for care and use of laboratory animals.

\section{Microinjections}

Capped synthetic mRNAs generated using mMessage mMachine kit (Ambion) were dissolved in nuclease free water and injected into embryos immersed in 3\% Ficoll using standard methods (Sive et al., 2000). Each injection delivered between $\sim 3 \mathrm{~nL}$ or $\sim 3-4 \mathrm{ng}$ of mRNA (per blastomere) into the embryos, usually at 4-cell stage into the middle of one or more cells (in the animal pole) as indicated in the Results section. Constructs used was Kv1.5 (Strutz-Seebohm et al., 2007) and Kv1.5-2A-b-galactosidase, both in vector PCS2. Kv1.5-2A-b-galactosidase was injected as a bi-cistronic RNA (with single polyA tail at the end) which produces separate proteins, due to a viral peptide sequence $(2 A)$ inserted between the 2 cDNA sequences (de Felipe et al., 2006, Szymczak-Workman et al., 2012). Kv1.5 is a commonly-used hyperpolarizing channel (Bertoli et al., 1994, Pai et al., 2012a, Strutz-Seebohm et al., 2007).

\section{Immunofluorescence and immunohistochemistry}

Spatial detection of apoptosis and proliferation was performed by immunofluorescence for activated caspase 3 and phospho-histone H3P respectively, on sections. Stage 30 embryos were used because by this stage, the brain region of the neural tube is specified and the brain morphology is now developing, making it a good stage to assess apoptosis and proliferation within the developing brain. Briefly, embryos were fixed overnight in MEMFA at 4 degrees (Sive et al., 2000), embedded in agarose and sectioned at $100 \mu \mathrm{m}$ thickness using a Leica vibratome (VT1000S) as per standard protocol (Blackiston et al., 2010) or embedded in paraffin and sectioned at $5 \mu \mathrm{m}$ thickness using Leica microtome as per the standard protocol (Sive et al., 2000). The sections [ $>5$ sections per embryo $(n=$ number of embryos as indicated in the results)] were permeabilized in PBS $0.1 \%$ Triton X-100 (PBST), antigen retrieved using citrate buffer $(\mathrm{pH}$ 6.0) (heating in a microwave), blocked with $10 \%$ goat serum in PBST for 1 hour at room temperature and incubated at $4^{\circ} \mathrm{C}$ overnight with primary antibody (Apoptosis - Anti-Active Caspase 3; Abcam ab13847) (Proliferation -Anti-H3P; Millipore-Invitrogen 04-817) at 1:1000 dilution in PBST+10\% goat serum (blocking buffer). Sections were washed six times in PBST and incubated with Alexa-Fluor conjugated fluorescent secondary antibody (Invitrogen) at 1:500 dilution in PBST $+10 \%$ goat serum overnight at $4{ }^{\circ} \mathrm{C}$. Sections were washed six times in PBST and photographed using Olympus BX-61 microscope equipped with a Hamamatsu ORCA AG CCD camera, and controlled by Metamorph software.

\section{Drug exposure}

Xenopus embryos were incubated in pharmacological blockers dissolved in 0.1X MMR during the stages of interest as indicated in respective experiments followed by several washes with 0.1X MMR. Embryos were exposed (from stage 10 - stage 30 unless otherwise specified, because neural/brain tissue development takes place in this time period, allowing specific testing of effects on these processes while allowing cleavage and gastrulation to proceed normally) to: $20 \mu \mathrm{M}$ Apoptosis inhibitor M50054 (CalbioChem 178488).

\section{Beta-galactosidase staining}

Embryos injected with $\beta$-galmRNA were fixed at gastrula or neurula stages, washed, and stained with X-gal (Roche Applied Sciences, Indianapolis, IN) at $37^{\circ} \mathrm{C}$.

\section{Statistics}

All statistical analysis was performed using Microsoft Excel. Data was either pooled from various iterations, with $\chi^{2}$-Square analysis performed on them, or data from various iterations was analyzed by $t$-test (for 2 groups) or ANOVA (for more than two groups).

\section{Acknowledgements}

We thank Erin Switzer and Amanda Allen for Xenopus husbandry and general lab assistance, Dany Adams for help with microscopy, and Florian Lang for Kv1.5 plasmid, we gratefully acknowledge support of the NIH (AR055993-01), NSF (CBET-0939511), and the G. Harold and Leila Y. Mathers Charitable Foundation. The authors declare no competing financial interests.

\section{References}

ADAMS, D.J. and LEVIN, M. (2006). Strategies and techniques for investigation of biophysical signals in patterning. In Analysis of growth factor signaling in embryos (Eds. M. Whitman and A.K . Sater) CRC Press, pp. 177-262.

ADAMS, D.S. and LEVIN, M. (2013). Endogenous voltage gradients as mediators of 
cell-cell communication: strategies for investigating bioelectrical signals during pattern formation. Cell Tissue Res 352: 95-122.

ADAMS, D.S., MASI, A. and LEVIN, M. (2007). H+ pump-dependent changes in membrane voltage are an early mechanism necessary and sufficient to induce Xenopus tail regeneration. Development 134: 1323-1335.

ALTMANN, C.R. and BRIVANLOU, A.H. (2001). Neural patterning in the vertebrate embryo. Int Rev Cytol 203: 447-482.

ANAVA, S., SAAD, Y. and AYALI, A. (2013). The role of gap junction proteins in the development of neural network functional topology. Insect Mol Biol 22: 457-472.

ARYA, R. and WHITE, K. (2015). Cell death in development: Signaling pathways and core mechanisms. Semin Cell Dev Biol. 39:12-19. (doi: 10.1016/j. semcdb.2015.02.001)

AW, S., ADAMS, D.S., QIU, D. and LEVIN, M. (2008). H,K-ATPase protein localization and Kir4.1 function reveal concordance of three axes during early determination of left-right asymmetry. Mech Dev 125: 353-372.

AW, S., KOSTER, J.C., PEARSON, W., NICHOLS, C.G., SHI, N.Q., CARNEIRO, K. and LEVIN, M. (2010). The ATP-sensitive K(+)-channel (K(ATP)) controls early left-right patterning in Xenopus and chick embryos. Dev Biol 346: 39-53.

BEANE, W.S., MOROKUMA, J., ADAMS, D.S. and LEVIN, M. (2011). A chemical genetics approach reveals $\mathrm{H}, \mathrm{K}$-ATPase-mediated membrane voltage is required for planarian head regeneration. Chem Biol 18: 77-89.

BEANE, W.S., MOROKUMA, J., LEMIRE, J.M. and LEVIN, M. (2013). Bioelectric signaling regulates head and organ size during planarian regeneration. Development 140: 313-322.

BERGMANN, A. and STELLER, H. (2010). Apoptosis, stem cells, and tissue regeneration. Sci Signal 3: re8.

BERTOLI, A., MORAN, O. and CONTI, F. (1994). Activation and deactivation properties of rat brain K+ channels of the Shaker-related subfamily. EurBiophys J23:379-384.

BINGGELI, R. and WEINSTEIN, R. (1986a). Membrane potentials and sodium channels: hypotheses for growth regulation and cancer formation based on changes in sodium channels and gap junctions. J. Theor. Biol. 123: 377-401.

BINGGELI, R. and WEINSTEIN, R.C. (1986b). Membrane potentials and sodium channels: hypotheses for growth regulation and cancer formation based on changes in sodium channels and gap junctions. J Theor Biol 123: 377-401.

BLACKISTON, D., ADAMS, D.S., LEMIRE, J.M., LOBIKIN, M. and LEVIN, M. (2011). Transmembrane potential of GlyCl-expressing instructor cells induces a neoplastic-like conversion of melanocytes via a serotonergic pathway. Dis Model Mech 4: 67-85.

BLACKISTON, D., VANDENBERG, L.N. and LEVIN, M. (2010). High-Throughput Xenopus laevis Immunohistochemistry Using Agarose Sections. Cold Spring Harb Protoc 2010: pdb prot5532.

BLACKISTON, D.J., ANDERSON, G.M., RAHMAN, N., BIECK, C. and LEVIN, M. (2015a). A Novel Method for Inducing Nerve Growth via Modulation of Host Resting Potential: Gap Junction-Mediated and Serotonergic Signaling Mechanisms. Neurotherapeutics 12: 170-184.

BLACKISTON, D.J., ANDERSON, G.M., RAHMAN, N., BIECK, C. and LEVIN, M. (2015b). A Novel Method for Inducing Nerve Growth via Modulation of Host Resting Potential: Gap Junction-Mediated and Serotonergic Signaling Mechanisms. Neurotherapeutics 12: 170-184

BLACKISTON, D.J., MCLAUGHLIN, K.A. and LEVIN, M. (2009). Bioelectric controls of cell proliferation: ion channels, membrane voltage and the cell cycle. Cell Cycle 8: 3519-3528.

BORODINSKY, L.N., ROOT, C.M., CRONIN, J.A., SANN, S.B., GU, X. and SPITZER, N.C. (2004). Activity-dependent homeostatic specification of transmitter expression in embryonic neurons. Nature 429: 523-530.

BRACKENBURY, W.J. (2012). Voltage-gated sodium channels and metastatic disease. Channels (Austin) 6: 352-61.

BURR, H.S. (1932). An electro-dynamic theory of development suggested by studies of proliferation rates in the brain of Amblystoma. J. Comp. Neurol. 56: 347-371.

CAO, L., WEI, D., REID, B., ZHAO, S., PU, J., PAN, T., YAMOAH, E. and ZHAO, M. (2013). Endogenous electric currents might guide rostral migration of neuroblasts. EMBO Rep 14: 184-190.

CHAN, W.Y., LORKE, D.E., TIU, S.C. and YEW, D.T. (2002). Proliferation and apoptosis in the developing human neocortex. Anat Rec 267: 261-276.

CHERA, S., GHILA, L., DOBRETZ, K., WENGER, Y., BAUER, C., BUZGARIU, W.,
MARTINOU, J.C. and GALLIOT, B. (2009). Apoptotic cells provide an unexpected source of Wnt3 signaling to drive hydra head regeneration. Dev Cell 17: 279-289.

CHERNET, B.T., FIELDS, C. and LEVIN, M. (2014). Long-range gap junctional signaling controls oncogene-mediated tumorigenesis in Xenopus laevis embryos. Front Physiol 5: 519

CHERNET, B.T. and LEVIN, M. (2013a). Transmembrane voltage potential is an essential cellular parameter for the detection and control of tumor development in a Xenopus model. Dis Model Mech 6: 595-607.

CHERNET, B.T. and LEVIN, M. (2013b). Transmembrane voltage potential is an essential cellular parameter for the detection and control of tumor development in a Xenopus model. Dis Model Mech 6: 595-607.

CHERNET, B.T. and LEVIN, M. (2014). Transmembrane voltage potential of somatic cells controls oncogene-mediated tumorigenesis at long-range. Oncotarget 5 3287-306.

COPP, A.J. and GREENE, N.D. (2010). Genetics and development of neural tube defects. J Pathol 220: 217-230.

COWAN, C.M. and ROSKAMS, A.J. (2004). Caspase-3 and caspase-9 mediate developmental apoptosis in the mouse olfactory system. J Comp Neurol 474: 136-148.

DE FELIPE, P., LUKE, G.A., HUGHES, L.E., GANI, D., HALPIN, C. and RYAN, M.D. (2006). E unum pluribus: multiple proteins from a self-processing polyprotein. Trends Biotechnol 24: 68-75.

DEISSEROTH, K., SINGLA, S., TODA, H., MONJE, M., PALMER, T.D. and MALENKA R.C. (2004). Excitation-neurogenesis coupling in adult neural stem/progenitor cells. Neuron 42: 535-552.

DETRAIT, E.R., GEORGE, T.M., ETCHEVERS, H.C., GILBERT, J.R., VEKEMANS M. and SPEER, M.C. (2005). Human neural tube defects: developmental biology, epidemiology, and genetics. Neurotoxicol Teratol 27: 515-524.

DING, F., ZHANG, G., LIU, L., JIANG, L., WANG, R., ZHENG, Y., WANG, G., XIE M. and DUAN, Y. (2012). Involvement of cationic channels in proliferation and migration of human mesenchymal stem cells. Tissue \& Cell 44: 358-364.

DOGANLI, C., BECK, H.C., RIBERA, A.B., OXVIG, C. and LYKKE-HARTMANN, K. (2013). alpha3Na+/K+-ATPase deficiency causes brain ventricle dilation and abrupt embryonic motility in zebrafish. J Biol Chem 288: 8862-8874.

FUHRMANN, S. Eye morphogenesis and patterning of the optic vesicle. Curr Top Dev Biol 93: 61-84.

FUKUMOTO, T., BLAKELY, R. and LEVIN, M. (2005). Serotonin transporter function is an early step in left-right patterning in chick and frog embryos. Dev Neurosci 27: 349-363.

GOLDMAN, S., O'BRIEN, L.M., FILIPEK, P.A., RAPIN, I. and HERBERT, M.R. (2013) Motor stereotypies and volumetric brain alterations in children with Autistic Disorder Res Autism Spectr Disord 7: 82-92.

GOTZ, M. and HUTTNER, W.B. (2005). The cell biology of neurogenesis. Nat Rev Mol Cell Biol 6: 777-788.

HARADA, T., HARADA, C. and PARADA, L.F. (2007). Molecular regulation of visual system development: more than meets the eye. Genes Dev 21: 367-378.

HARVEY, K.F. and HARIHARAN, I.K. (2012). The hippo pathway. Cold Spring Harb Perspect Biol 4: a011288.

HIGASHIMORI, H. and SONTHEIMER, H. (2007). Role of Kir4.1 channels in growth control of glia. Glia 55: 1668-1679.

JAFFE, L.F. (1979). Control of development by ionic currents. Soc Gen Physiol Ser 33: 199-231.

JOSEPH, B. and HERMANSON, O. (2010). Molecular control of brain size: regulators of neural stem cell life, death and beyond. Exp Cell Res 316: 1415-1421.

KONIG, S., HINARD, V., ARNAUDEAU, S., HOLZER, N., POTTER, G., BADER, C.R. and BERNHEIM, L. (2004). Membrane hyperpolarization triggers myogenin and myocyte enhancer factor-2 expression during human myoblast differentiation. $J$ Biol Chem 279: 28187-28196.

KOZOROVITSKIY, Y., SAUNDERS, A., JOHNSON, C.A., LOWELL, B.B. and SABATINI, B.L. (2012). Recurrent network activity drives striatal synaptogenesis. Nature 485: 646-650.

KROLL, K.L. and AMAYA, E. (1996). Transgenic Xenopus embryos from sperm nuclear transplantations reveal FGF signaling requirements during gastrulation. Development 122: 3173-3183.

LAUDER, J.M., WALLACE, J.A. and KREBS, H. (1981). Roles for serotonin in neuroembryogenesis. Adv Exp Med Biol 133: 477-506. 
LEVIN, M. (2009). Errors of geometry: regeneration in a broader perspective. Sem. Cell Dev. Biol. 20: 643-645.

LEVIN, M. (2013a). Reprogramming cells and tissue patterning via bioelectrical pathways: molecular mechanisms and biomedical opportunities. Wiley Interdiscip Rev Syst Biol Med 5: 657-676.

LEVIN, M. (2013b). Reprogramming cells and tissue patterning via bioelectrical pathways: molecular mechanisms and biomedical opportunities. Wiley Interdiscip Rev Syst Biol Med 5: 657-676.

LEVIN, M. (2014a). Endogenous bioelectrical networks store non-genetic patterning information during development and regeneration. J. Physiol. 592: 2295-2305.

LEVIN, M. (2014b). Molecular bioelectricity: how endogenous voltage potentials control cell behavior and instruct pattern regulation in vivo. Mol. Biol. Cell25: 3835-3850.

LEVIN, M. and MERCOLA, M. (1999). Gap junction-mediated transfer of left-right patterning signals in the early chick blastoderm is upstream of Shh asymmetry in the node. Development 126: 4703-4714.

LEVIN, M. and STEVENSON, C.G. (2012). Regulation of cell behavior and tissue patterning by bioelectrical signals: challenges and opportunities for biomedical engineering. Annu Rev Biomed Eng 14: 295-323.

LEVIN, M., THORLIN, T., ROBINSON, K.R., NOGI, T. and MERCOLA, M. (2002). Asymmetries in $\mathrm{H}+/ \mathrm{K}+-A T P a s e$ and cell membrane potentials comprise a very early step in left-right patterning. Cell 111: 77-89.

LIN, G., CHEN, Y. and SLACK, J.M. (2012). Transgenic analysis of signaling pathways required for Xenopus tadpole spinal cord and muscle regeneration. Anat Rec. 295: 1532-1540.

LOBIKIN, M., CHERNET, B., LOBO, D. and LEVIN, M. (2012a). Resting potential, oncogene-induced tumorigenesis, and metastasis: the bioelectric basis of cancer in vivo. Phys Biol 9: 065002.

LOBIKIN, M., CHERNET, B., LOBO, D. and LEVIN, M. (2012b). Resting potential, oncogene-induced tumorigenesis, and metastasis: the bioelectric basis of cancer in vivo. Phys Biol 9: 065002.

MARSH, G. and BEAMS, H.W. (1947). Electrical control of growth polarity in regenerating Dugesia tigrina. Fed Proc 6: 163.

MARSH-ARMSTRONG, N., HUANG, H., BERRY, D.L. and BROWN, D.D. (1999). Germ-line transmission of transgenes in Xenopus laevis. Proc Natl Acad Sci USA 96: 14389-14393.

MCCAIG, C.D., RAJNICEK, A.M., SONG, B. and ZHAO, M. (2005). Controlling cell behavior electrically: current views and future potential. Physiol Rev 85: 943-978.

MIURA, M. (2011). Active participation of cell death in development and organismal homeostasis. Dev Growth Differ 53: 125-136.

MOODY, S.A. (1987). Fates of the blastomeres of the 32-cell-stage Xenopus embryo. Dev Biol 122: 300-319.

MUSTARD, J. and LEVIN, M. (2014). Bioelectrical Mechanisms for Programming Growth and Form: Taming Physiological Networks for Soft Body Robotics. Soft Robotics 1: 169-191.

NG, S.Y., CHIN, C.H., LAU, Y.T., LUO, J., WONG, C.K., BIAN, Z.X. and TSANG, S.Y. (2010). Role of voltage-gated potassium channels in the fate determination of embryonic stem cells. J Cell Physiol 224: 165-177.

NIEUWKOOP, P.D. and FABER, J. (1967). Normal Table of Xenopus laevis (Daudin). North-Holland Publishing Company, Amsterdam.

NILIUS, B. and WOHLRAB, W. (1992). Potassium channels and regulation of proliferation of human melanoma cells. J Physiol 445: 537-548.

NONOMURA, K., YAMAGUCHI, Y., HAMACHI, M., KOIKE, M., UCHIYAMA, Y., NAKAZATO, K., MOCHIZUKI, A., SAKAUE-SAWANO, A., MIYAWAKI, A., YOSHIDA, H. et al., (2013). Local apoptosis modulates early mammalian brain development through the elimination of morphogen-producing cells. Dev Cell 27: 621-634.

NORTHCUTT, R.G. (1996). The Agnathan ark: the origin of craniate brains. Brain Behav Evol 48: 237-247.

NORTHCUTT, R.G. (2003). Origin of the isthmus? A comparison of the brains of lancelets and vertebrates. J Comp Neurol 466: 316-318.

NUCKELS, R.J., NG, A., DARLAND, T. and GROSS, J.M. (2009). The vacuolarATPase complex regulates retinoblast proliferation and survival, photoreceptor morphogenesis, and pigmentation in the zebrafish eye. Invest Ophthalmol Vis Sci 50: 893-905.

PAI, V., LEMIRE, J.M., PARE, J.F., LIN, G., CHEN, Y. and LEVIN, M. (2015). Endogenous gradients of resting potential instructively pattern embryonic neural tissue via Notch signaling and regulation of proliferation. J. Neuroscience 35: 4366-85 (doi: 10.1523/JNEUROSCI.1877-14.2015)

PAI, V.P., AW, S., SHOMRAT, T., LEMIRE, J.M. and LEVIN, M. (2012a). Transmembrane voltage potential controls embryonic eye patterning in Xenopus laevis. Development 139: 313-323.

PAI, V.P. and LEVIN, M. (2014). Chapter 5: Bioelectric Control of Stem Cell Function. In Stem Cells: From Basic Research to Therapy, vol. 1 (ed. CALEGARI, F. and WASKOV, C.). Science Publisher, pp.106-148.

PAI, V.P., VANDENBERG, L.N., BLACKISTON, D. and LEVIN, M. (2012b). Neurally Derived Tissues in Xenopus laevis Embryos Exhibit a Consistent Bioelectrical Left-Right Asymmetry. Stem Cells Int 2012: 353491.

PALACIOS-PRADO, N. and BUKAUSKAS, F.F. (2009). Heterotypic gap junction channels as voltage-sensitive valves for intercellular signaling. Proc Natl Acad Sci USA 106: 14855-14860

PENN, A.A. and SHATZ, C.J. (1999). Brain waves and brain wiring: the role of endogenous and sensory-driven neural activity in development. Pediatr Res 45: 447-458.

PERATHONER, S., DAANE, J.M., HENRION, U., SEEBOHM, G., HIGDON, C.W., JOHNSON, S.L., NUSSLEIN-VOLHARD, C. and HARRIS, M.P. (2014). Bioelectric signaling regulates size in zebrafish fins. PLoS Genet 10: e1004080.

PEREZ-GARIJO, A., FUCHS, Y. and STELLER, H. (2013). Apoptotic cells can induce non-autonomous apoptosis through the TNF pathway. eLife 2: e01004.

PORTER, A.G. and JANICKE, R.U. (1999). Emerging roles of caspase-3 in apoptosis. Cell Death Differ 6: 99-104.

PRATT, K.G. and KHAKHALIN, A.S. (2013). Modeling human neurodevelopmental disorders in the Xenopus tadpole: from mechanisms to therapeutic targets. Dis Model Mech 6: 1057-1065

RAKIC, S. and ZECEVIC, N. (2000). Programmed cell death in the developing human telencephalon. Eur J Neurosci 12: 2721-2734.

RICHARD B. BORGENS, K.R.R., JOSEPH W. VANABLE, JR., AND MICHAEL E. MCGINNIS, WITH COLIN D. MCCAIG. (1989). Electric Fields in Vertebrate Repair: Natural and Applied Voltages in Vertebrate Regeneration and Healing. Wiley-Liss, New York.

SAKA, Y. and SMITH, J.C. (2001). Spatial and temporal patterns of cell division during early Xenopus embryogenesis. Dev Biol 229: 307-318.

SANCHEZALVARADO, A. (2003). The freshwater planarian Schmidtea mediterranea: embryogenesis, stem cells and regeneration. Curr Opin Genet Dev 13: 438-444.

SHITAMUKAI, A. and MATSUZAKI, F. (2012). Control of asymmetric cell division of mammalian neural progenitors. Dev Growth Differ 54: 277-286.

SIVE, H.L., GRAINGER, R.M. and HARLAND, R.M. (2000). Early Development of Xenopus laevis. Cold Spring Harbor Laboratory Press, New York.

STANGER, B.Z. (2008a). The biology of organ size determination. Diabetes Obes Metab 10 Suppl 4: 16-22.

STANGER, B.Z. (2008b). Organ size determination and the limits of regulation. Cell Cycle 7: 318-324.

STRUTZ-SEEBOHM, N., GUTCHER, I., DECHER, N., STEINMEYER, K., LANG F. and SEEBOHM, G. (2007). Comparison of potent Kv1.5 potassium channel inhibitors reveals the molecular basis for blocking kinetics and binding mode. Cell Physiol Biochem 20: 791-800.

SUNDELACRUZ, S., LEVIN, M. and KAPLAN, D.L. (2008). Membrane potential controls adipogenic and osteogenic differentiation of mesenchymal stem cells. PLOS ONE 3: e3737.

SUNDELACRUZ, S., LEVIN, M. and KAPLAN, D.L. (2009). Role of membrane potential in the regulation of cell proliferation and differentiation. Stem Cell Rev 5: 231-246.

SUNDELACRUZ, S., LI, C., CHOI, Y.J., LEVIN, M. and KAPLAN, D.L. (2013). Bioelectric modulation of wound healing in a $3 \mathrm{D}$ in vitro model of tissue-engineered bone. Biomaterials 34: 6695-6705.

SWAPNA, I. and BORODINSKY, L.N. (2012). Interplay between electrical activity and bone morphogenetic protein signaling regulates spinal neuron differentiation Proc Natl Acad Sci USA 109: 16336-16341.

SZYMCZAK-WORKMAN, A.L., VIGNALI, K.M. and VIGNALI, D.A. (2012). Design and construction of $2 \mathrm{~A}$ peptide-linked multicistronic vectors. Cold Spring Harb Protoc 2012: 199-204.

TANNAHILL, D., HARRIS, L.W. and KEYNES, R. (2005). Role of morphogens in brain growth. J Neurobiol 64: 367-375. 
THOMPSON, D. (1942). On Growith and Form. Cambridge University Press, Cambridge, England.

TSENG, A. and LEVIN, M. (2013a). Cracking the bioelectric code: Probing endogenous ionic controls of pattern formation. Commun Integr Biol 6: e22595.

TSENG, A. and LEVIN, M. (2013b). Cracking the bioelectric code: Probing endogenous ionic controls of pattern formation. Commun Integr Biol 6: 1-8.

TSENG, A.S., ADAMS, D.S., QIU, D., KOUSTUBHAN, P. and LEVIN, M. (2007). Apoptosis is required during early stages of tail regeneration in Xenopus laevis. Dev Biol 301: 62-69.

TSENG, A.S., BEANE, W.S., LEMIRE, J.M., MASI, A. and LEVIN, M. (2010). Induction of vertebrate regeneration by a transient sodium current. $J$ Neurosci 30: $13192-13200$.

TSUDA, T., OHMORI, Y., MURAMATSU, H., HOSAKA, Y., TAKIGUCHI, K., SAITOH, F., KATO, K., NAKAYAMA, K., NAKAMURA, N., NAGATA, S. et al., (2001). Inhibitory effect of M50054, a novel inhibitor of apoptosis, on anti-Fas-antibody-induced hepatitis and chemotherapy-induced alopecia. Eur J Pharmacol 433: 37-45.

VAN VLIET, P., DE BOER, T.P., VANDER HEYDEN, M.A., ELTAMER, M.K., SLUIJTER, J.P., DOEVENDANS, P.A. and GOUMANS, M.J. (2010). Hyperpolarization induces differentiation in human cardiomyocyte progenitor cells. Stem Cell Rev6:178-185.

VANDENBERG, L.N., MORRIE, R.D. andADAMS, D.S. (2011). V-ATPase-dependent ectodermal voltage and $\mathrm{pH}$ regionalization are required for craniofacial morpho- genesis. Dev Dyn 240: 1889-1904.

VERSELIS, V.K., BENNETT, M.V. and BARGIELLO, T.A. (1991). A voltage-dependent gap junction in Drosophila melanogaster. Biophysical J. 59: 114-126.

WALLINGFORD, J.B. (2006). Planar cell polarity, ciliogenesis and neural tube defects. Human Molec. Genet. 15 Spec No 2: R227-R234.

WARNER, A.E. (1985). The role of gap junctions in amphibian development. J Embryol Exp Morphol 89 Suppl: 365-380.

YANG, M. and BRACKENBURY, W.J. (2013). Membrane potential and cancer progression. Front. Physiol. 4: 185.

ZHANG, J., CHAN, Y.C., HO, J.C., SIU, C.W., LIAN, Q. and TSE, H.F. (2012). Regulation of Cell Proliferation of Human Induced Pluripotent Stem Cell-Derived Mesenchymal Stem Cells via Ether a go-go 1 (hEAG1) Potassium Channel. Am. J. Physiol. Cell Physiol. 303: C115-25. (doi: 10.1152/ajpcell.00326.2011)

ZHAO, B., TUMANENG, K. and GUAN, K.L. (2011). The Hippo pathway in organ size control, tissue regeneration and stem cell self-renewal. Nat Cell Biol 13: 877-883.

ZHAO, M., SONG, B., PU, J., WADA, T., REID, B., TAI, G., WANG, F., GUO, A., WALCZYSKO, P., GU, Y. et al., (2006). Electrical signals control wound healing through phosphatidylinositol-3-OH kinase-gamma and PTEN. Nature 442: 457-460.

ZUBER, M.E. (2010). Eye field specification in Xenopus laevis. Curr Top Dev Biol 93: $29-60$. 


\section{Further Related Reading, published previously in the Int. J. Dev. Biol.}

Visualization, characterization and modulation of calcium signaling during the development of slow muscle cells in intact zebrafish embryos

Chris Y. Cheung, Sarah E. Webb, Donald R. Love and Andrew L. Miller

Int. J. Dev. Biol. (2011) 55: 153-174

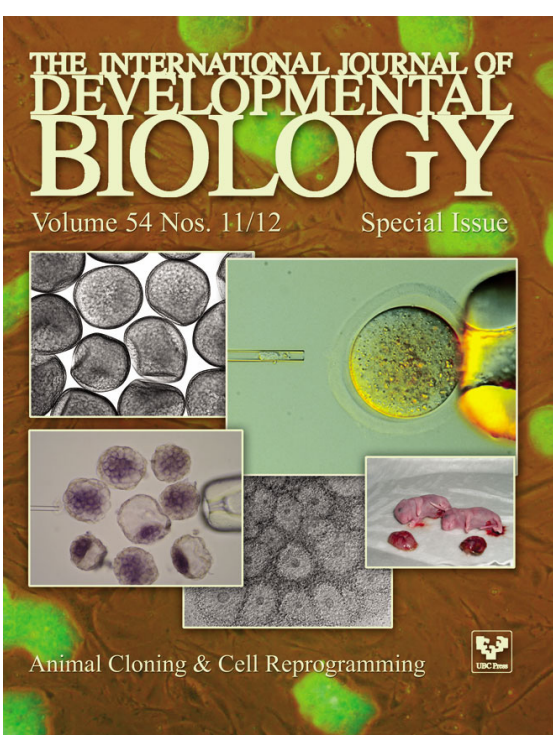

Oscillatory $\mathrm{Ca} 2+$ dynamics and cell cycle resumption at fertilization in mammals: a modelling approach

Geneviève Dupont, Elke Heytens and Luc Leybaert

Int. J. Dev. Biol. (2010) 54: 655-665

The role of ion fluxes in polarized cell growth and morphogenesis: the pollen tube as an experimental paradigm

Erwan Michard, Filipa Alves and José A. Feijó

Int. J. Dev. Biol. (2009) 53: 1609-1622

Sperm-activating peptides in the regulation of ion fluxes, signal transduction and motility Alberto Darszon, Adán Guerrero, Blanca E. Galindo, Takuya Nishigaki and Christopher D. Wood Int. J. Dev. Biol. (2008) 52: 595-606

The dynamics of calcium oscillations that activate mammalian eggs Karl Swann and Yuansong Yu

Int. J. Dev. Biol. (2008) 52: 585-594

The choice between epidermal and neural fate: a matter of calcium. Marc Moreau and Catherine Leclerc Int. J. Dev. Biol. (2004) 48: 75-84
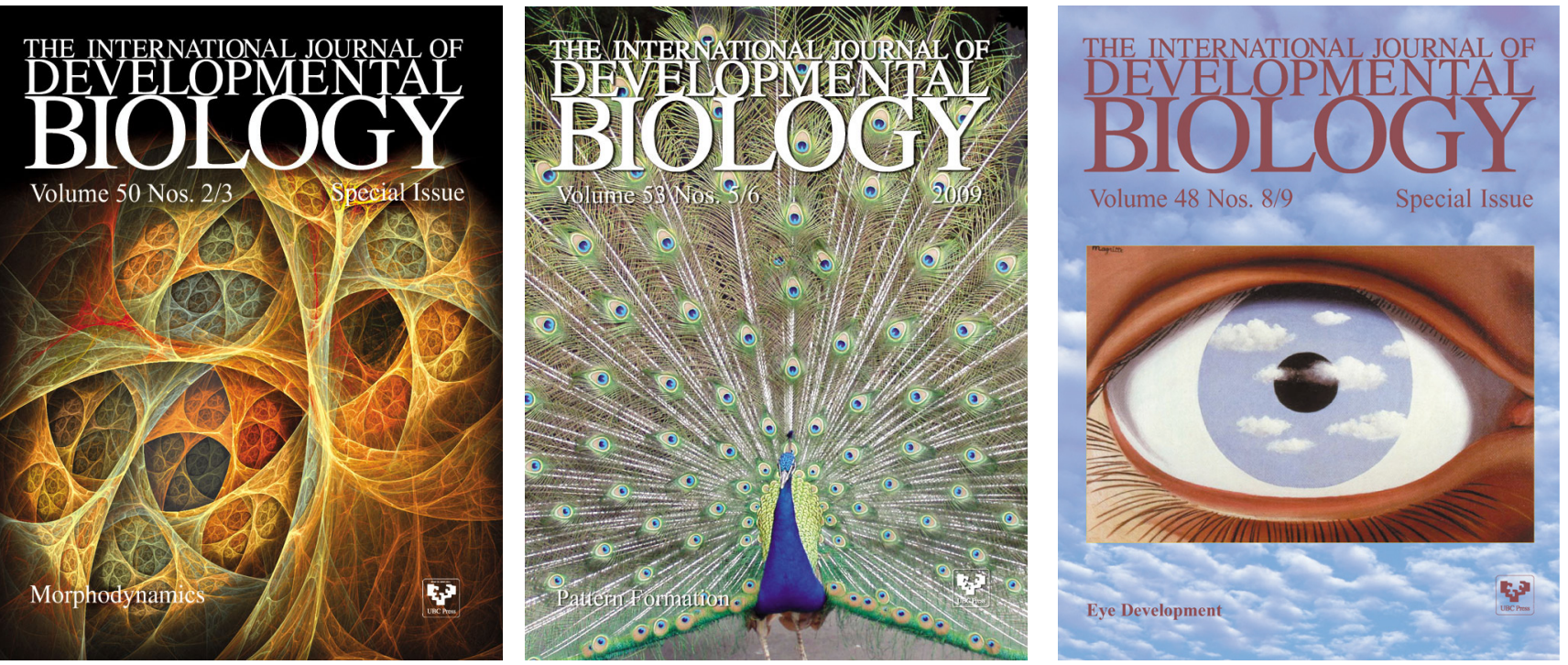